MATHEMATICS OF COMPUTATION

Volume 78, Number 266, April 2009, Pages 645-671

S $0025-5718(08) 02176-5$

Article electronically published on October 17, 2008

\title{
ERROR ANALYSIS FOR THE ELASTIC FLOW OF PARAMETRIZED CURVES
}

\author{
KLAUS DECKELNICK AND GERHARD DZIUK
}

\begin{abstract}
We analyze a semidiscrete numerical scheme for approximating the evolution of parametric curves by elastic flow in $\mathbb{R}^{n}$. The fourth order equation is split into two coupled second order problems, which are approximated by linear finite elements. We prove error bounds for the resulting scheme and present numerical test calculations that confirm our analysis.
\end{abstract}

\section{INTRODUCTION}

Let $x:[0,2 \pi] \rightarrow \mathbb{R}^{n}(n \geq 2)$ be the parametrization of a closed curve. For $\lambda>0$ we consider the functional

$$
E_{\lambda}(x):=\frac{1}{2} \int_{0}^{2 \pi} \kappa^{2} d s+\lambda L(x)
$$

where $\kappa$ denotes the curvature of the curve and $L(x)$ is its length. Furthermore, $d s$ is the arclength element. The first part in $E_{\lambda}$ is the well-known bending energy; in view of its scaling properties, it is common to penalize length giving rise to the second term in (1.1). The critical points of $E_{\lambda}$ are called elasticae. A natural way to obtain stable elasticae is to consider the limits as $t \rightarrow \infty$ of the gradient flow associated with $E_{\lambda}$ which is given by the following evolution equation

$$
x_{t}=-\nabla_{s s}^{2} y-\frac{1}{2}|y|^{2} y+\lambda y
$$

where $y=x_{s s}$ is the curvature vector and $\nabla_{s} f=f_{s}-\left(f_{s}, \tau\right) \tau, \tau=x_{s}$ denoting the unit tangent. Obviously the velocity $x_{t}$ has no tangential component.

For $n=2$, Polden 6 proved the global existence of smooth solutions for (1.2). The corresponding result for curves in arbitrary codimension was obtained in [5]. That paper also suggests a numerical method in order to calculate approximate solutions of (1.2). A different scheme was used in 1 for the length preserving elastic flow. A special feature of their approach is that it introduces a tangential motion which leads to good numerical properties of the scheme. However, up to now there is no error analysis for the two above-mentioned methods. The purpose of this paper is to introduce a new finite element scheme, which respects the variational structure of the problem, and to carry out an error analysis.

The elastic flow for surfaces is called the Willmore flow. Error estimates for the Willmore flow of two-dimensional graphs have been obtained by the authors in

Received by the editor November 5, 2007 and, in revised form, April 10, 2008.

2000 Mathematics Subject Classification. Primary 35K55, 65M15, 65M60.

This work was supported by the Deutsche Forschungsgemeinschaft via DFG-Forschergruppe 469 Nonlinear partial differential equations: Theoretical and numerical analysis.

(c) 2008 American Mathematical Society Reverts to public domain 28 years from publication 
2. The underlying evolution equation for the height function is a nonlinear strictly parabolic PDE of fourth order. In contrast, the equation (1.2) is a nonlinear system of fourth order which is in addition degenerate in a tangential direction. This degeneracy is due to certain invariance properties of the operator and complicates the analysis. A numerical scheme for the Willmore flow of two-dimensional surfaces is presented in 4 for the semidiscrete problem.

For a survey over numerical methods for geometric PDEs we refer to 3 .

Notation. We denote by $\|\cdot\|_{L^{p}}(1 \leq p \leq \infty)$ and $\|\cdot\|_{H^{1}}$ the norm of $L^{p}(0,2 \pi)$ and $H^{1}(0,2 \pi)$, respectively. For $p=2$ we write $\|\cdot\|_{L^{2}}=\|\cdot\|$. Finally, $(\cdot, \cdot)$ is the euclidean scalar product in $\mathbb{R}^{n}$.

\section{VARIATIONAL FORMULATION AND DISCRETIZATION}

Let us begin by deriving a formula for the first variation of $E_{\lambda}$ which we shall use in order to deduce a suitable variational form for (1.2). The curves $x=x(u)$ to be considered are defined on $[0,2 \pi]$ and are in general not parametrized by arclength so that we have

$$
\tau=\frac{x_{u}}{\left|x_{u}\right|}, \quad y=\frac{1}{\left|x_{u}\right|}\left(\frac{x_{u}}{\left|x_{u}\right|}\right)_{u}=\frac{1}{\left|x_{u}\right|} \tau_{u} .
$$

Thus,

$$
E_{\lambda}(x)=\frac{1}{2} \int_{0}^{2 \pi}|y|^{2}\left|x_{u}\right|+\lambda \int_{0}^{2 \pi}\left|x_{u}\right|
$$

and hence for all $\phi \in H_{\text {per }}^{1}\left((0,2 \pi), \mathbb{R}^{n}\right)$,

$$
\left\langle E_{\lambda}^{\prime}(x), \phi\right\rangle=\int_{0}^{2 \pi}\left(y, y_{\phi}\right)\left|x_{u}\right|+\frac{1}{2} \int_{0}^{2 \pi}|y|^{2}\left(\tau, \phi_{u}\right)+\lambda \int_{0}^{2 \pi}\left(\tau, \phi_{u}\right),
$$

where $y_{\phi}$ denotes the derivative of $y$ in direction $\phi$ and $H_{\text {per }}^{1}\left((0,2 \pi), \mathbb{R}^{n}\right)$ is the space of all periodic functions in $H^{1}\left((0,2 \pi), \mathbb{R}^{n}\right)$. In order to calculate $y_{\phi}$ we write the relation between $x$ and its curvature vector $y$ in variational form, i.e.

$$
\int_{0}^{2 \pi}(y, \psi)\left|x_{u}\right|+\int_{0}^{2 \pi}\left(\tau, \psi_{u}\right)=0 \quad \forall \psi \in H_{\text {per }}^{1}\left((0,2 \pi), \mathbb{R}^{n}\right) .
$$

As a consequence for all $\psi \in H_{\text {per }}^{1}\left((0,2 \pi), \mathbb{R}^{n}\right)$,

$$
\int_{0}^{2 \pi}\left(y_{\phi}, \psi\right)\left|x_{u}\right|+\int_{0}^{2 \pi}(y, \psi)\left(\tau, \phi_{u}\right)+\int_{0}^{2 \pi} \frac{1}{\left|x_{u}\right|}\left(P \phi_{u}, \psi_{u}\right)=0,
$$

where $P$ is the projection matrix $P=I_{n}-\tau \otimes \tau$. Using $\psi=y$ in the above identity then gives

$$
\left\langle E_{\lambda}^{\prime}(x), \phi\right\rangle=-\int_{0}^{2 \pi} \frac{1}{\left|x_{u}\right|}\left(P y_{u}, \phi_{u}\right)-\frac{1}{2} \int_{0}^{2 \pi}|y|^{2}\left(\tau, \phi_{u}\right)+\lambda \int_{0}^{2 \pi}\left(\tau, \phi_{u}\right) .
$$

It is therefore natural to introduce the following weak form of the gradient flow for the functional $E_{\lambda}$ :

$$
\begin{array}{r}
\int_{0}^{2 \pi}\left(x_{t}, \phi\right)\left|x_{u}\right|-\int_{0}^{2 \pi} \frac{\left(P y_{u}, \phi_{u}\right)}{\left|x_{u}\right|}-\frac{1}{2} \int_{0}^{2 \pi}|y|^{2}\left(\tau, \phi_{u}\right)+\lambda \int_{0}^{2 \pi}\left(\tau, \phi_{u}\right)=0 \\
\int_{0}^{2 \pi}(y, \psi)\left|x_{u}\right|+\int_{0}^{2 \pi}\left(\tau, \psi_{u}\right)=0
\end{array}
$$


for all $\phi, \psi \in H_{\mathrm{per}}^{1}\left((0,2 \pi), \mathbb{R}^{n}\right)$ and $0 \leq t \leq T$. It is not difficult to verify that for a smooth function $x$ (2.2), (2.3) is equivalent to (1.2).

We now use (2.2), (2.3) in order to define our numerical scheme. Let $0=u_{0}<$ $u_{1}<\cdots<u_{N-1}<u_{N}=2 \pi$ be a partition of $[0,2 \pi]$ into subintervals $I_{j}=\left[u_{j-1}, u_{j}\right]$ and $h_{j}:=u_{j}-u_{j-1}$ as well as $h:=\max _{j=1, \ldots, N} h_{j}$. We require the following inverse assumption

$$
h \leq \hat{c} h_{j} \quad \text { for all } j=1, \ldots, N,
$$

where $\hat{c}$ is independent of $h$. Let us denote by $X_{h}$ the space of linear finite elements, i.e.

$$
\begin{aligned}
X_{h}=\left\{\eta_{h} \in C^{0}([0,2 \pi]) \mid \eta_{h \mid I_{j}} \text { is a linear polynomial, } j\right. & =1, \ldots, N \\
\text { and } \eta_{h}(0) & \left.=\eta_{h}(2 \pi)\right\}
\end{aligned}
$$

with the usual nodal basis $\left\{\varphi_{1}, \ldots, \varphi_{N}\right\}$. In order to deal with vector-valued functions we also introduce $X_{h}^{n}:=\left\{\phi_{h}:[0,2 \pi] \rightarrow \mathbb{R}^{n} \mid \phi_{h, i} \in X_{h}, i=1, \ldots, n\right\}$. We denote by $I_{h}$ the Lagrange interpolation operator,

$$
I_{h} f=\sum_{j=1}^{N} f\left(u_{j}\right) \varphi_{j}
$$

for which we have the standard interpolation estimates

$$
\left\|f-I_{h} f\right\|+h\left\|f_{u}-\left(I_{h} f\right)_{u}\right\| \leq C h^{2}\|f\|_{H^{2}} \quad \forall f \in H_{\text {per }}^{2}(0,2 \pi) .
$$

Furthermore, the following properties will be useful:

$$
\begin{aligned}
\int_{I_{j}}\left|\phi_{h}\right|^{2} & \leq \int_{I_{j}} I_{h}\left[\left|\phi_{h}\right|^{2}\right] \leq C \int_{I_{j}}\left|\phi_{h}\right|^{2} \\
\int_{I_{j}}\left(\phi_{h}, \psi_{h}\right) & =\int_{I_{j}} I_{h}\left[\left(\phi_{h}, \psi_{h}\right)\right]-\frac{1}{6} h_{j}^{2} \int_{I_{j}}\left(\phi_{h u}, \psi_{h u}\right)
\end{aligned}
$$

for $j=1, \ldots, N$ and all $\phi_{h}, \psi_{h} \in X_{h}^{n}$. Let us finally introduce

$$
Z_{h}:=\left\{z_{h}:[0,2 \pi] \rightarrow \mathbb{R}^{n} \mid z_{h \mid I_{j}} \text { is constant, } j=1, \ldots, N\right\}
$$

and define $Q_{h}: H^{1}\left((0,2 \pi), \mathbb{R}^{n}\right) \rightarrow Z_{h}$ by $\left(Q_{h} f\right)_{\mid I_{j}}:=\frac{1}{\left|I_{j}\right|} \int_{I_{j}} f, \quad j=1, \ldots, N$. Clearly,

$$
\left\|f-Q_{h} f\right\| \leq C h\|f\|_{H^{1}}, \quad \forall f \in H^{1}\left((0,2 \pi), \mathbb{R}^{n}\right) .
$$

The semi-discrete problem corresponding to (2.2), (2.3) can then be stated as follows: find $x_{h}, y_{h}:[0,2 \pi] \times[0, T] \rightarrow \mathbb{R}^{n}$ such that $x_{h}(\cdot, t), y_{h}(\cdot, t) \in X_{h}^{n}, 0 \leq t \leq T$ and

$$
\begin{aligned}
& \int_{0}^{2 \pi} I_{h}\left[\left(x_{h t}, \phi_{h}\right)\right]\left|x_{h u}\right|-\int_{0}^{2 \pi} \frac{\left(P_{h} y_{h u}, \phi_{h u}\right)}{\left|x_{h u}\right|}-\frac{1}{2} \int_{0}^{2 \pi} I_{h}\left[\left|y_{h}\right|^{2}\right]\left(\tau_{h}, \phi_{h u}\right) \\
& +\lambda \int_{0}^{2 \pi}\left(\tau_{h}, \phi_{h u}\right)=0 \\
& \int_{0}^{2 \pi} I_{h}\left[\left(y_{h}, \psi_{h}\right)\right]\left|x_{h u}\right|+\int_{0}^{2 \pi}\left(\tau_{h}, \psi_{h u}\right)=0 \\
& x_{h}(\cdot, 0)=I_{h} x_{0}
\end{aligned}
$$


for all $\phi_{h}, \psi_{h} \in X_{h}^{n}$ and $0 \leq t \leq T$. Here, we have used the abbreviations

$$
\tau_{h}=\frac{x_{h u}}{\left|x_{h u}\right|}, \quad P_{h}=I_{n}-\tau_{h} \otimes \tau_{h} .
$$

Remark 2.1. a ) In view of (2.3), (2.10) the terms $\lambda \int_{0}^{2 \pi}\left(\tau, \phi_{u}\right)$ and $\lambda \int_{0}^{2 \pi}\left(\tau_{h}, \phi_{h u}\right)$ can be replaced by $-\lambda \int_{0}^{2 \pi}(y, \phi)\left|x_{u}\right|$ and $-\lambda \int_{0}^{2 \pi} I_{h}\left[\left(y_{h}, \phi_{h}\right)\right]\left|x_{h u}\right|$, respectively.

b) By inserting $\phi_{h}=x_{h t}$ into (2.9), $\psi_{h}=y_{h}$ into (2.10) differentiated with respect to time and observing (3.5), (3.6) below, we immediately obtain the following energy decrease as long as the discrete solution exists:

$$
\int_{0}^{2 \pi} I_{h}\left[\left|x_{h t}\right|^{2}\right]\left|x_{h u}\right|+\frac{d}{d t}\left\{\frac{1}{2} \int_{0}^{2 \pi} I_{h}\left[\left|y_{h}\right|^{2}\right]\left|x_{h u}\right|+\lambda \int_{0}^{2 \pi}\left|x_{h u}\right|\right\}=0 .
$$

An analagous testing procedure will be at the heart of our error analysis.

Note that our choice of the initial datum in (2.11) determines $y_{h}(\cdot, 0) \in X_{h}^{n}$ through the relation

$$
\begin{aligned}
\int_{0}^{2 \pi} I_{h}\left[\left(y_{h}(\cdot, 0), \psi_{h}\right)\right]\left|\left(I_{h} x_{0}\right)_{u}\right| & \\
& +\int_{0}^{2 \pi} \frac{\left(\left(I_{h} x_{0}\right)_{u}, \psi_{h u}\right)}{\left|\left(I_{h} x_{0}\right)_{u}\right|}=0 \quad \text { for all } \psi_{h} \in X_{h}^{n} .
\end{aligned}
$$

The following result shows that $y_{h}(\cdot, 0)$ is a good approximation of $y(\cdot, 0)$.

Lemma 2.2. Suppose that $x_{h 0}=I_{h} x_{0}$. Then for $h \leq h_{0}$,

$$
\left\|y(\cdot, 0)-y_{h}(\cdot, 0)\right\| \leq C h .
$$

Proof. Recalling (2.12), (2.7) and the relation $y(\cdot, 0)=\frac{1}{\left|x_{0 u}\right|}\left(\frac{x_{0 u}}{\left|x_{0 u}\right|}\right)_{u}$ we obtain

$$
\begin{aligned}
\int_{0}^{2 \pi} & I_{h}\left[\left(I_{h} y(\cdot, 0)-y_{h}(\cdot, 0), \psi_{h}\right)\right]\left|\left(I_{h} x_{0}\right)_{u}\right| \\
& =\int_{0}^{2 \pi}\left(I_{h} y(\cdot, 0)-y(\cdot, 0), \psi_{h}\right)\left|\left(I_{h} x_{0}\right)_{u}\right| \\
& +\int_{0}^{2 \pi}\left(y(\cdot, 0), \psi_{h}\right)\left(\left|\left(I_{h} x_{0}\right)_{u}\right|-\left|x_{0 u}\right|\right)+\int_{0}^{2 \pi}\left(\frac{\left(I_{h} x_{0}\right)_{u}}{\left|\left(I_{h} x_{0}\right)_{u}\right|}-\frac{x_{0 u}}{\left|x_{0 u}\right|}, \psi_{h u}\right) \\
& +\frac{1}{6} \sum_{j=1}^{N} h_{j}^{2} \int_{I_{j}}\left(\left(I_{h} y(\cdot, 0)\right)_{u}, \psi_{h u}\right)\left|\left(I_{h} x_{0}\right)_{u}\right| \\
& \equiv \sum_{j=1}^{4} S_{j}
\end{aligned}
$$

for all $\psi_{h} \in X_{h}^{n}$. Clearly, (2.5) and an inverse estimate implies that

$$
\left|S_{1}\right|+\left|S_{2}\right|+\left|S_{4}\right| \leq C h\left\|\psi_{h}\right\|,
$$

while

$$
S_{3}=\sum_{j=1}^{N}\left(\psi_{h u \mid I_{j}}, e_{j}\right), e_{j}=h_{j}\left(\frac{x_{0}\left(u_{j}\right)-x_{0}\left(u_{j-1}\right)}{\left|x_{0}\left(u_{j}\right)-x_{0}\left(u_{j-1}\right)\right|}-\tau_{0}\left(m_{j}\right)\right)+\left(h_{j} \tau_{0}\left(m_{j}\right)-\int_{I_{j}} \tau_{0} d u\right)
$$


with $\tau_{0}=\frac{x_{0 u}}{\left|x_{0 u}\right|}$ and $m_{j}=\left(u_{j-1}+u_{j}\right) / 2$. The error formula for the midpoint rule and a Taylor expansion yield $\left|e_{j}\right| \leq C h_{j}^{3}$ and hence

$$
\left|S_{3}\right| \leq C h\left\|\psi_{h}\right\|
$$

again by an inverse inequality. The result now follows by setting $\psi_{h}=I_{h} y(\cdot, 0)-$ $y_{h}(\cdot, 0)$.

Our main results are the following error bounds:

Theorem 2.3. Let $x:[0,2 \pi] \times[0, \infty) \rightarrow \mathbb{R}^{n}$ be the solution of (1.2) with smooth initial value $x(\cdot, 0)=x_{0}$ and $T>0$. Then there exists $h_{0}>0$ such that (2.9) -(2.11) has a unique solution on $[0, T]$ and

$$
\begin{gathered}
\sup _{t \in[0, T]}\left\|x(\cdot, t)-x_{h}(\cdot, t)\right\|_{H^{1}}^{2}+\int_{0}^{T}\left\|x_{t}(\cdot, t)-x_{h t}(\cdot, t)\right\|^{2} d t \leq C h^{2}, \\
\sup _{t \in[0, T]}\left\|y(\cdot, t)-y_{h}(\cdot, t)\right\|^{2}+\int_{0}^{T}\left\|y_{u}(\cdot, t)-y_{h u}(\cdot, t)\right\|^{2} d t \leq C h^{2}
\end{gathered}
$$

for all $0<h \leq h_{0}$. The constant $C$ depends on $T$, $\inf _{(0,2 \pi)}\left|x_{0 u}\right|$ and on higher norms of the solution $x$ of the continuous problem.

In order not to overburden the presentation and in view of the global existence results for the continuous problem, we do not trace the precise norms of the continuous solution.

The proof of the theorem will be carried out in $\S 3$. It turns out that in order to gain control on the first derivatives of $x$ it is necessary to deal separately with the directions and the lengths of $x_{u}, x_{h u}$, respectively. This is due to the degeneracy of the PDE in the tangential direction.

\section{ERROR ANALYSIS}

Let us fix $T>0$. In view of the smoothness of the continuous solution there exist constants $0<c_{0} \leq C_{0}$ such that

$$
c_{0} \leq\left|x_{u}\right| \leq C_{0}, \quad|y| \leq C_{0} \text { in }[0,2 \pi] \times[0, T], \quad \int_{0}^{T}\left\|y_{u}\right\|_{L^{\infty}}^{2} d t \leq C_{0} .
$$

Standard ODE theory implies that (2.9)-(2.11) has a unique solution $\left(x_{h}, y_{h}\right)$ on some time interval $\left[0, T_{h}\right]\left(T_{h}>0\right)$ such that

$$
\frac{1}{2} c_{0} \leq\left|x_{h u}\right| \leq 2 C_{0},\left|y_{h}\right| \leq 2 C_{0} \text { in }[0,2 \pi] \times\left[0, T_{h}\right], \int_{0}^{T_{h}}\left\|y_{h u}\right\|_{L^{\infty}}^{2} d t \leq 2 C_{0}
$$

Let us define

$$
\begin{aligned}
\hat{T}_{h} & :=\sup \left\{t \in[0, T] \mid\left(x_{h}, y_{h}\right) \text { solves (2.9)-(2.11) on }[0, t]\right. \text { and } \\
& \left.\frac{1}{2} c_{0} \leq\left|x_{h u}\right| \leq 2 C_{0}, \quad\left|y_{h}\right| \leq 2 C_{0} \text { in }[0,2 \pi] \times[0, t], \quad \int_{0}^{t}\left\|y_{h u}\right\|_{L^{\infty}}^{2} d t \leq 2 C_{0}\right\} .
\end{aligned}
$$

Our aim is to show that $\hat{T}_{h}=T$ for small $h$. To this purpose we shall prove the bounds (2.13) and (2.14) on $\left[0, \hat{T}_{h}\right]$ with constants only depending on $T, c_{0}, C_{0}$ and 
norms of the continuous solution allowing us to continue the discrete solution. By the definition of $\hat{T}_{h}$ we have

$$
\frac{1}{2} c_{0} \leq\left|x_{h u}\right| \leq 2 C_{0},\left|y_{h}\right| \leq 2 C_{0} \text { on }[0,2 \pi] \times\left[0, \hat{T}_{h}\right), \int_{0}^{\hat{T}_{h}}\left\|y_{h u}\right\|_{L^{\infty}}^{2} d t \leq 2 C_{0}
$$

For later use we note some useful identities:

$$
\begin{aligned}
(v, w) & =1-\frac{1}{2}|v-w|^{2} \quad \text { for } v, w \in \mathbb{R}^{n},|v|=|w|=1 \\
\left|x_{u}\right|_{t} & =\left(x_{t u}, \tau\right),\left|x_{h u}\right|_{t}=\left(x_{h t u}, \tau_{h}\right) \\
\tau_{t} & =\frac{1}{\left|x_{u}\right|} P x_{t u}, \tau_{h t}=\frac{1}{\left|x_{h u}\right|} P_{h} x_{h t u} .
\end{aligned}
$$

We split the error analysis into several steps starting with two lemmas that provide the basic estimate.

\section{Lemma 3.1.}

$$
\begin{aligned}
& \frac{c_{0}}{4}\left\|x_{t}-x_{h t}\right\|^{2}-\int_{0}^{2 \pi}\left(\frac{1}{\left|x_{u}\right|} P y_{u}-\frac{1}{\left|x_{h u}\right|} P_{h} y_{h u}, x_{t u}-x_{h t u}\right) \\
& -\frac{1}{2} \int_{0}^{2 \pi}\left(|y|^{2} \tau-\left|y_{h}\right|^{2} \tau_{h}, x_{t u}-x_{h t u}\right) \leq C\left(h^{2}+\left\|y-y_{h}\right\|_{H^{1}}^{2}+\left\|\left|x_{u}\right|-\left|x_{h u}\right|\right\|^{2}\right) .
\end{aligned}
$$

Proof. Inserting $\phi_{h}=I_{h} x_{t}-x_{h t}$ in (2.2) and (2.9), taking the difference of the resulting identities and recalling Remark 2.1] as well as (2.7) we infer

$$
\begin{aligned}
& \int_{0}^{2 \pi}\left|x_{t}-x_{h t}\right|^{2}\left|x_{h u}\right|-\int_{0}^{2 \pi}\left(\frac{1}{\left|x_{u}\right|} P y_{u}-\frac{1}{\left|x_{h u}\right|} P_{h} y_{h u},\left(I_{h} x_{t}\right)_{u}-x_{h t u}\right) \\
& \text { (3.7) } \quad-\frac{1}{2} \int_{0}^{2 \pi}\left(|y|^{2} \tau-\left|y_{h}\right|^{2} \tau_{h},\left(I_{h} x_{t}\right)_{u}-x_{h t u}\right)=\int_{0}^{2 \pi}\left(x_{t}-I_{h} x_{t}, x_{t}-x_{h t}\right)\left|x_{h u}\right| \\
& +\lambda \int_{0}^{2 \pi}\left(y-y_{h}, I_{h} x_{t}-x_{h t}\right)\left|x_{h u}\right|+\lambda \int_{0}^{2 \pi}\left(y, I_{h} x_{t}-x_{h t}\right)\left(\left|x_{u}\right|-\left|x_{h u}\right|\right) \\
& +\frac{1}{6} \sum_{j=1}^{N} h_{j}^{2} \int_{I_{j}}\left(\left(x_{h t u},\left(I_{h} x_{t}\right)_{u}-x_{h t u}\right)\left|x_{h u}\right|-\frac{1}{2}\left|y_{h u}\right|^{2}\left(\tau_{h},\left(I_{h} x_{t}\right)_{u}-x_{h t u}\right)\right. \\
& \left.-\lambda\left(y_{h u},\left(I_{h} x_{t}\right)_{u}-x_{h t u}\right)\left|x_{h u}\right|\right)+\int_{0}^{2 \pi}\left(x_{t}, I_{h} x_{t}-x_{h t}\right)\left(\left|x_{h u}\right|-\left|x_{u}\right|\right) \\
& \equiv \sum_{i=1}^{5} S_{i}
\end{aligned}
$$

We deduce from (2.5) and (3.3) that

$$
\sum_{i \neq 4} S_{i} \leq \frac{1}{2} \int_{0}^{2 \pi}\left|x_{t}-x_{h t}\right|^{2}\left|x_{h u}\right|+C\left(h^{4}+\left\|y-y_{h}\right\|^{2}+\left\|\left|x_{u}\right|-\left|x_{h u}\right|\right\|^{2}\right) .
$$


On the other hand, an inverse estimate, (3.3), Young's inequality and (2.5) imply

$$
\begin{array}{rl}
S_{4} \leq & \frac{1}{6} \sum_{j=1}^{N} h_{j}^{2} \int_{I_{j}}\left\{-\left|\left(I_{h} x_{t}\right)_{u}-x_{h t u}\right|^{2}+\left(\left(I_{h} x_{t}\right)_{u},\left(I_{h} x_{t}\right)_{u}-x_{h t u}\right)\right\}\left|x_{h u}\right| \\
& +C \sum_{j=1}^{N} \int_{I_{j}}\left(h_{j}^{2}\left|\left(I_{h} y\right)_{u}\right|^{2}+h_{j}\left|\left(I_{h} y\right)_{u}-y_{h u}\right|+h_{j}^{2}\right)\left|\left(I_{h} x_{t}\right)_{u}-x_{h t u}\right| \\
9 & C h^{2}+C\left\|y_{u}-y_{h u}\right\|^{2} .
\end{array}
$$

Furthermore, since

$$
\int_{0}^{2 \pi}\left(x_{t u}, z_{h}\right)=\int_{0}^{2 \pi}\left(\left(I_{h} x_{t}\right)_{u}, z_{h}\right) \quad \forall z_{h} \in Z_{h},
$$

we obtain, with the help of (2.5) and (2.8),

$$
\begin{gathered}
\int_{0}^{2 \pi}\left(\frac{1}{\left|x_{u}\right|} P y_{u}-\frac{1}{\left|x_{h u}\right|} P_{h} y_{h u},\left(I_{h} x_{t}\right)_{u}\right)=\int_{0}^{2 \pi}\left(\frac{1}{\left|x_{u}\right|} P y_{u}-\frac{1}{\left|x_{h u}\right|} P_{h} y_{h u}, x_{t u}\right) \\
+\int_{0}^{2 \pi}\left(\frac{1}{\left|x_{u}\right|} P y_{u}-Q_{h}\left[\frac{1}{\left|x_{u}\right|} P y_{u}\right],\left(I_{h} x_{t}\right)_{u}-x_{t u}\right) \\
\leq \int_{0}^{2 \pi}\left(\frac{1}{\left|x_{u}\right|} P y_{u}-\frac{1}{\left|x_{h u}\right|} P_{h} y_{h u}, x_{t u}\right)+C h^{2}
\end{gathered}
$$

and similarly

$$
\frac{1}{2} \int_{0}^{2 \pi}\left(|y|^{2} \tau-\left|y_{h}\right|^{2} \tau_{h},\left(I_{h} x_{t}\right)_{u}\right) \leq \frac{1}{2} \int_{0}^{2 \pi}\left(|y|^{2} \tau-\left|y_{h}\right|^{2} \tau_{h}, x_{t u}\right)+C h^{2}+C\left\|y-y_{h}\right\|^{2} .
$$

The result now follows after inserting the above inequalities as well as (3.8), (3.9) into (3.7) and observing (3.3).

Lemma 3.2. We have for $\epsilon>0$,

$$
\begin{aligned}
\frac{1}{2} \frac{d}{d t} \int_{0}^{2 \pi} I_{h}\left[\left|I_{h} y-y_{h}\right|^{2} \mid\right]\left|x_{h u}\right|+\int_{0}^{2 \pi}\left(\frac{1}{\left|x_{u}\right|} P x_{t u}-\frac{1}{\left|x_{h u}\right|} P_{h} x_{h t u}, y_{u}-y_{h u}\right) \\
\quad-\frac{1}{2} \int_{0}^{2 \pi}\left|y-y_{h}\right|^{2}\left(x_{h t u}, \tau_{h}\right)+\int_{0}^{2 \pi}\left(\left(x_{t u}, \tau\right) y-\left(x_{h t u}, \tau_{h}\right) y_{h}, y-y_{h}\right) \\
\leq \epsilon\left\|x_{t}-x_{h t}\right\|^{2}+C_{\epsilon}\left(h^{2}+\left\|y-y_{h}\right\|_{H^{1}}^{2}+\left\|\tau-\tau_{h}\right\|^{2}+\left\|\left|x_{u}\right|-\left|x_{h u}\right|\right\|^{2}\right) .
\end{aligned}
$$

Proof. We differentiate (2.3) and (2.10) with respect to time; in view of (3.6) and (2.7), we obtain

$$
\begin{aligned}
\int_{0}^{2 \pi} I_{h} & {\left[\left(I_{h} y_{t}-y_{h t}, \psi_{h}\right)\right]\left|x_{h u}\right|+\int_{0}^{2 \pi}\left(\frac{1}{\left|x_{u}\right|} P x_{t u}-\frac{1}{\left|x_{h u}\right|} P_{h} x_{h t u}, \psi_{h u}\right) } \\
& +\int_{0}^{2 \pi}\left(\left(x_{t u}, \tau\right) y-\left(x_{h t u}, \tau_{h}\right) y_{h}, \psi_{h}\right) \\
= & \int_{0}^{2 \pi}\left(I_{h} y_{t}-y_{t}, \psi_{h}\right)\left|x_{h u}\right|+\int_{0}^{2 \pi}\left(y_{t}, \psi_{h}\right)\left(\left|x_{h u}\right|-\left|x_{u}\right|\right) \\
& +\frac{1}{6} \sum_{j=1}^{N} h_{j}^{2} \int_{I_{j}}\left(\left(I_{h} y_{t}\right)_{u}, \psi_{h u}\right)\left|x_{h u}\right|+\frac{1}{6} \sum_{j=1}^{N} h_{j}^{2} \int_{I_{j}}\left(y_{h u}, \psi_{h u}\right)\left(x_{h t u}, \tau_{h}\right)
\end{aligned}
$$


for all $\psi_{h} \in X_{h}^{n}$. If we choose $\psi_{h}=I_{h} y-y_{h}$ in (3.10) we obtain

$$
\begin{aligned}
\frac{1}{2} \frac{d}{d t} \int_{0}^{2 \pi} I_{h}\left[\left|I_{h} y-y_{h}\right|^{2}\right]\left|x_{h u}\right|+\int_{0}^{2 \pi}\left(\frac{1}{\left|x_{u}\right|} P x_{t u}-\frac{1}{\left|x_{h u}\right|} P_{h} x_{h t u},\left(I_{h} y\right)_{u}-y_{h u}\right) \\
\quad+\int_{0}^{2 \pi}\left(\left(x_{t u}, \tau\right) y-\left(x_{h t u}, \tau_{h}\right) y_{h}, I_{h} y-y_{h}\right)-\frac{1}{2} \int_{0}^{2 \pi}\left|I_{h} y-y_{h}\right|^{2}\left(x_{h t u}, \tau_{h}\right) \\
=\int_{0}^{2 \pi}\left(I_{h} y_{t}-y_{t}, I_{h} y-y_{h}\right)\left|x_{h u}\right|+\int_{0}^{2 \pi}\left(y_{t}, I_{h} y-y_{h}\right)\left(\left|x_{h u}\right|-\left|x_{u}\right|\right) \\
\quad+\frac{1}{6} \sum_{j=1}^{N} h_{j}^{2} \int_{I_{j}}\left\{\left(y_{h u},\left(I_{h} y\right)_{u}-y_{h u}\right)+\frac{1}{2}\left|\left(I_{h} y\right)_{u}-y_{h u}\right|^{2}\right\}\left(x_{h t u}, \tau_{h}\right) \\
\quad+\frac{1}{6} \sum_{j=1}^{N} h_{j}^{2} \int_{I_{j}}\left(\left(I_{h} y_{t}\right)_{u},\left(I_{h} y\right)_{u}-y_{h u}\right)\left|x_{h u}\right| \equiv \sum_{i=1}^{4} \tilde{S}_{i} .
\end{aligned}
$$

We infer from (2.5) and (3.3)

$$
\left|\tilde{S}_{1}\right|+\left|\tilde{S}_{2}\right| \leq C\left(h^{4}+\left\|y-y_{h}\right\|^{2}+\left\|\left|x_{u}\right|-\left|x_{h u}\right|\right\|^{2}\right),
$$

while an inverse estimate along with (3.3) implies

$$
\begin{aligned}
\left|\tilde{S}_{3}\right| & \leq C h^{2}\left(\left\|y_{h u}\right\|_{L^{\infty}}+\left\|\left(I_{h} y\right)_{u}\right\|_{L^{\infty}}\right)\left\|\left(I_{h} y\right)_{u}-y_{h u}\right\|\left\|x_{h t u}\right\| \\
& \leq C h\left(\left\|y_{h}\right\|_{L^{\infty}}+\|y\|_{L^{\infty}}\right)\left\|\left(I_{h} y\right)_{u}-y_{h u}\right\|\left(\left\|\left(I_{h} x_{t}\right)_{u}-x_{h t u}\right\|+\left\|\left(I_{h} x_{t}\right)_{u}\right\|\right) \\
& \leq C\left\|\left(I_{h} y\right)_{u}-y_{h u}\right\|\left(\left\|I_{h} x_{t}-x_{h t}\right\|+h\right) \\
& \leq \epsilon\left\|x_{t}-x_{h t}\right\|^{2}+C_{\epsilon}\left(h^{2}+\left\|y_{u}-y_{h u}\right\|^{2}\right) .
\end{aligned}
$$

Using again an inverse inequality we obtain

$$
\left|\tilde{S}_{4}\right| \leq C h^{2}\left\|\left(I_{h} y_{t}\right)_{u}\right\|\left\|\left(I_{h} y\right)_{u}-y_{h u}\right\| \leq C\left(h^{2}+\left\|y-y_{h}\right\|^{2}\right) .
$$

As in the proof of Lemma 3.1 we have

$$
\begin{aligned}
& \int_{0}^{2 \pi}\left(\frac{1}{\left|x_{u}\right|} P x_{t u}-\frac{1}{\left|x_{h u}\right|} P_{h} x_{h t u},\left(I_{h} y\right)_{u}\right) \\
& \geq \int_{0}^{2 \pi}\left(\frac{1}{\left|x_{u}\right|} P x_{t u}-\frac{1}{\left|x_{h u}\right|} P_{h} x_{h t u}, y_{u}\right)-C h^{2}
\end{aligned}
$$

Furthermore, a straightforward calculation shows

$$
\begin{aligned}
- & \frac{1}{2}\left|I_{h} y-y_{h}\right|^{2}\left(x_{h t u}, \tau_{h}\right)+\left(\left(x_{t u}, \tau\right) y-\left(x_{h t u}, \tau_{h}\right) y_{h}, I_{h} y-y_{h}\right) \\
= & -\frac{1}{2}\left|y-y_{h}\right|^{2}\left(x_{h t u}, \tau_{h}\right)+\left(\left(x_{t u}, \tau\right) y-\left(x_{h t u}, \tau_{h}\right) y_{h}, y-y_{h}\right) \\
& \quad-\frac{1}{2}\left|y-I_{h} y\right|^{2}\left(x_{h t u}, \tau_{h}\right)-\left[\left(x_{t u}-x_{h t u}, \tau\right)+\left(x_{h t u}, \tau-\tau_{h}\right)\right]\left(y, y-I_{h} y\right) .
\end{aligned}
$$


Similarly as above we estimate

$$
\begin{aligned}
\mid \int_{0}^{2 \pi}( & \left(-\frac{1}{2}\left|y-I_{h} y\right|^{2}\left(x_{h t u}, \tau_{h}\right)-\left[\left(x_{t u}-x_{h t u}, \tau\right)+\left(x_{h t u}, \tau-\tau_{h}\right)\right]\left(y, y-I_{h} y\right)\right) \mid \\
\leq & C\left\|y-I_{h} y\right\|_{L^{\infty}}\left(\left\|y-I_{h} y\right\|+\left\|\tau-\tau_{h}\right\|\right)\left\|x_{h t u}\right\|+\left\|x_{t u}-x_{h t u}\right\|\left\|y-I_{h} y\right\| \\
\leq & C h^{2}\left(h^{2}+\left\|\tau-\tau_{h}\right\|\right)\left(\left\|x_{h t u}-\left(I_{h} x_{t}\right)_{u}\right\|+\left\|\left(I_{h} x_{t}\right)_{u}\right\|\right) \\
& +C h^{2}\left(\left\|x_{t u}-\left(I_{h} x_{t}\right)_{u}\right\|+\left\|\left(I_{h} x_{t}\right)_{u}-x_{h t u}\right\|\right) \\
\leq & \epsilon\left\|x_{t}-x_{h t}\right\|^{2}+C_{\epsilon}\left(h^{2}+\left\|\tau-\tau_{h}\right\|^{2}\right)
\end{aligned}
$$

and the assertion follows.

The next lemma combines the above results.

\section{Lemma 3.3.}

$\frac{c_{0}}{16}\left\|x_{t}-x_{h t}\right\|^{2}+\zeta^{\prime}(t) \leq C h^{2}+C\left(\left\|y-y_{h}\right\|_{H^{1}}^{2}+\left\|\tau-\tau_{h}\right\|^{2}+\left\|\left|x_{u}\right|-\left|x_{h u}\right|\right\|^{2}\right)$, where

$$
\begin{aligned}
\zeta(t)= & \frac{1}{2} \int_{0}^{2 \pi} I_{h}\left[\left|I_{h} y-y_{h}\right|^{2}\right]\left|x_{h u}\right|-\frac{1}{4} \int_{0}^{2 \pi}|y|^{2}\left|\tau-\tau_{h}\right|^{2}\left|x_{h u}\right| \\
& +\int_{0}^{2 \pi}\left(\frac{\left|x_{h u}\right|-\left|x_{u}\right|}{\left|x_{u}\right|}\left(\tau_{h}-\tau\right)+\frac{1}{2} \frac{\left|x_{h u}\right|}{\left|x_{u}\right|}\left|\tau_{h}-\tau\right|^{2} \tau, y_{u}\right) .
\end{aligned}
$$

Proof. It follows from Lemma 3.1 and Lemma 3.2 with $\epsilon=\frac{c_{0}}{8}$ that

$$
\begin{aligned}
& \frac{c_{0}}{8}\left\|x_{t}-x_{h t}\right\|^{2}+\frac{1}{2} \frac{d}{d t} \int_{0}^{2 \pi} I_{h}\left[\left|I_{h} y-y_{h}\right|^{2}\right]\left|x_{h u}\right|+\int_{0}^{2 \pi}(A+B) \\
& \leq C h^{2}+C\left(\left\|y-y_{h}\right\|_{H^{1}}^{2}+\left\|\tau-\tau_{h}\right\|^{2}+\left\|\left|x_{u}\right|-\left|x_{h u}\right|\right\|^{2}\right),
\end{aligned}
$$

where we have abbreviated

$$
\begin{aligned}
A= & -\frac{1}{2}\left(|y|^{2} \tau-\left|y_{h}\right|^{2} \tau_{h}, x_{t u}-x_{h t u}\right)-\frac{1}{2}\left|y-y_{h}\right|^{2}\left(x_{h t u}, \tau_{h}\right) \\
& +\left(\left(x_{t u}, \tau\right) y-\left(x_{h t u}, \tau_{h}\right) y_{h}, y-y_{h}\right) \\
B= & \left(\frac{1}{\left|x_{u}\right|} P x_{t u}-\frac{1}{\left|x_{h u}\right|} P_{h} x_{h t u}, y_{u}-y_{h u}\right) \\
& -\left(\frac{1}{\left|x_{u}\right|} P y_{u}-\frac{1}{\left|x_{h u}\right|} P_{h} y_{h u}, x_{t u}-x_{h t u}\right) .
\end{aligned}
$$

Let us rewrite the terms $A$ and $B$. To begin, a straightforward calculation shows that

$$
\begin{aligned}
A= & -\frac{1}{2}|y|^{2}\left(x_{h t u}, \tau_{h}\right)+\frac{1}{2}|y|^{2}\left(x_{h t u}, \tau\right)+\frac{1}{2}|y|^{2}\left(x_{t u}, \tau\right) \\
& +\frac{1}{2}\left|y_{h}\right|^{2}\left(x_{t u}, \tau_{h}\right)-\left(y, y_{h}\right)\left(x_{t u}, \tau\right) \\
= & \frac{1}{2}|y|^{2} \frac{\partial}{\partial t}\left(-\left|x_{h u}\right|+\left(x_{h u}, \tau\right)\right)-\frac{1}{2}|y|^{2}\left(x_{h u}, \tau_{t}\right)+\frac{1}{2}|y|^{2}\left(x_{t u}, \tau_{h}-\tau\right) \\
& +\frac{1}{2}\left|y-y_{h}\right|^{2}\left(x_{t u}, \tau_{h}\right)+\left(y, y-y_{h}\right)\left(x_{t u}, \tau-\tau_{h}\right) .
\end{aligned}
$$

Using (3.6), the symmetry of $P$ and (3.4) we obtain

$$
\left(x_{h u}, \tau_{t}\right)=\frac{\left|x_{h u}\right|}{\left|x_{u}\right|}\left(P \tau_{h}, x_{t u}\right)=\frac{\left|x_{h u}\right|}{\left|x_{u}\right|}\left(\tau_{h}-\tau, x_{t u}\right)+\frac{1}{2} \frac{\left|x_{h u}\right|}{\left|x_{u}\right|}\left(\tau, x_{t u}\right)\left|\tau_{h}-\tau\right|^{2} .
$$


In view of (3.4) we have $-\left|x_{h u}\right|+\left(x_{h u}, \tau\right)=-\frac{1}{2}\left|\tau-\tau_{h}\right|^{2}\left|x_{h u}\right|$ and hence

$$
\begin{aligned}
A & =-\frac{1}{4}|y|^{2} \frac{\partial}{\partial t}\left(\left|\tau_{h}-\tau\right|^{2}\left|x_{h u}\right|\right)+\frac{1}{2}|y|^{2}\left(1-\frac{\left|x_{h u}\right|}{\left|x_{u}\right|}\right)\left(\tau_{h}-\tau, x_{t u}\right) \\
& -\frac{1}{4}|y|^{2} \frac{\left|x_{h u}\right|}{\left|x_{u}\right|}\left(\tau, x_{t u}\right)\left|\tau_{h}-\tau\right|^{2}+\frac{1}{2}\left|y-y_{h}\right|^{2}\left(x_{t u}, \tau_{h}\right)+\left(y, y-y_{h}\right)\left(x_{t u}, \tau-\tau_{h}\right) .
\end{aligned}
$$

As a consequence we deduce

$$
\begin{aligned}
\int_{0}^{2 \pi} A \geq- & \frac{1}{4} \frac{d}{d t} \int_{0}^{2 \pi}|y|^{2}\left|\tau-\tau_{h}\right|^{2}\left|x_{h u}\right| \\
& -C\left(\left\|y-y_{h}\right\|^{2}+\left\|\tau-\tau_{h}\right\|^{2}+\left\|\left|x_{u}\right|-\left|x_{h u}\right|\right\|^{2}\right) .
\end{aligned}
$$

Next, recalling (3.6) we get

$$
\begin{aligned}
B= & \frac{1}{\left|x_{u}\right|}\left(y_{u}, P x_{h t u}\right)+\frac{1}{\left|x_{h u}\right|}\left(P_{h} y_{h u}, x_{t u}\right)-\frac{1}{\left|x_{u}\right|}\left(P x_{t u}, y_{h u}\right)-\frac{1}{\left|x_{h u}\right|}\left(P_{h} x_{h t u}, y_{u}\right) \\
= & \left(y_{u},\left[\frac{1}{\left|x_{u}\right|} P x_{h u}-\tau_{h}+\tau\right]_{t}\right)-\left(y_{u},\left(\frac{1}{\left|x_{u}\right|}\right)_{t} P x_{h u}+\frac{1}{\left|x_{u}\right|} P_{t} x_{h u}+\tau_{t}\right) \\
& +\left(x_{t u}, \frac{1}{\left|x_{h u}\right|} P_{h} y_{h u}-\frac{1}{\left|x_{u}\right|} P y_{h u}\right) .
\end{aligned}
$$

Combining the relation $P_{t}=-\tau \otimes \tau_{t}-\tau_{t} \otimes \tau$ with (3.5), (3.6) we have

$\left(\frac{1}{\left|x_{u}\right|}\right)_{t} P x_{h u}+\frac{1}{\left|x_{u}\right|} P_{t} x_{h u}=-\frac{\left|x_{h u}\right|}{\left|x_{u}\right|^{2}}\left(\tau, x_{t u}\right) P \tau_{h}-\frac{\left|x_{h u}\right|}{\left|x_{u}\right|^{2}}\left(\tau_{h}, \tau\right) P x_{t u}-\frac{\left|x_{h u}\right|}{\left|x_{u}\right|^{2}}\left(P \tau_{h}, x_{t u}\right) \tau$ and hence

$$
B=\left(y_{u},\left[\frac{1}{\left|x_{u}\right|} P x_{h u}-\tau_{h}+\tau\right]_{t}\right)+\left(x_{t u}, z_{1}\right)+\left(x_{t u}, z_{2}\right)
$$

where

$$
\begin{aligned}
z_{1} & =\frac{\left|x_{h u}\right|}{\left|x_{u}\right|^{2}}\left(\tau_{h}, \tau\right) P y_{u}-\frac{1}{\left|x_{u}\right|} P y_{u}-\frac{1}{\left|x_{u}\right|} P y_{h u}+\frac{1}{\left|x_{h u}\right|} P y_{h u}, \\
z_{2} & =\frac{\left|x_{h u}\right|}{\left|x_{u}\right|^{2}}\left(P \tau_{h}, y_{u}\right) \tau+\frac{\left|x_{h u}\right|}{\left|x_{u}\right|^{2}}\left(y_{u}, \tau\right) P \tau_{h}+\frac{1}{\left|x_{h u}\right|} P P_{h u}-\frac{1}{\left|x_{h u}\right|} P y_{h u} .
\end{aligned}
$$

Clearly,

$$
z_{1}=\frac{1}{\left|x_{h u}\right|}\left(\frac{\left|x_{h u}\right|}{\left|x_{u}\right|}-1\right)^{2} P y_{u}+\left(\left(\tau, \tau_{h}\right)-1\right) \frac{\left|x_{h u}\right|}{\left|x_{u}\right|^{2}} P y_{u}+\left(\frac{1}{\left|x_{u}\right|}-\frac{1}{\left|x_{h u}\right|}\right) P\left(y_{u}-y_{h u}\right)
$$

while

$$
\begin{aligned}
z_{2}= & \frac{\left|x_{h u}\right|}{\left|x_{u}\right|^{2}}\left(\left(\tau_{h}, y_{u}\right) \tau-2\left(\tau, \tau_{h}\right)\left(\tau, y_{u}\right) \tau+\left(\tau, y_{u}\right) \tau_{h}\right) \\
& +\frac{1}{\left|x_{h u}\right|}\left(\left(y_{h u}, \tau\right) \tau-\left(y_{h u}, \tau_{h}\right) \tau_{h}\right) \\
= & \left(y_{u}, \tau_{h}-\tau\right)\left(\frac{\left|x_{h u}\right|}{\left|x_{u}\right|^{2}} \tau-\frac{1}{\left|x_{h u}\right|} \tau_{h}\right)+\left(y_{u}, \tau\right)\left(\frac{\left|x_{h u}\right|}{\left|x_{u}\right|^{2}}-\frac{1}{\left|x_{h u}\right|}\right)\left(\tau_{h}-\tau\right) \\
& +2 \frac{\left|x_{h u}\right|}{\left|x_{u}\right|^{2}}\left(\tau, y_{u}\right)\left(1-\left(\tau, \tau_{h}\right)\right) \tau+\frac{1}{\left|x_{h u}\right|}\left(\left(y_{h u}-y_{u}, \tau\right) \tau-\left(y_{h u}-y_{u}, \tau_{h}\right) \tau_{h}\right) .
\end{aligned}
$$

Observing that

$$
\frac{1}{\left|x_{u}\right|} P x_{h u}-\tau_{h}+\tau=\left(\frac{\left|x_{h u}\right|}{\left|x_{u}\right|}-1\right)\left(\tau_{h}-\tau\right)+\frac{1}{2} \frac{\left|x_{h u}\right|}{\left|x_{u}\right|}\left|\tau-\tau_{h}\right|^{2} \tau
$$


and inserting the above form of $z_{1}, z_{2}$ into (3.13) and taking into account (3.4) we arrive at

$$
\begin{aligned}
\int_{0}^{2 \pi} B \geq & \frac{d}{d t} \int_{0}^{2 \pi}\left(\frac{\left|x_{h u}\right|-\left|x_{u}\right|}{\left|x_{u}\right|}\left(\tau_{h}-\tau\right)+\frac{1}{2} \frac{\left|x_{h u}\right|}{\left|x_{u}\right|}\left|\tau_{h}-\tau\right|^{2} \tau, y_{u}\right) \\
& -C\left(\left\|y_{u}-y_{h u}\right\|^{2}+\left\|\tau-\tau_{h}\right\|^{2}+\left\|\left|x_{u}\right|-\left|x_{h u}\right|\right\|^{2}\right) .
\end{aligned}
$$

Combining (3.11), (3.12) and (3.14) completes the proof of the lemma.

In order to proceed with the error analysis we have to deal with the terms $\left\|y_{u}-y_{h u}\right\|,\left\|\tau-\tau_{h}\right\|$ and $\left\|\left|x_{u}\right|-\left|x_{h u}\right|\right\|$ which appear on the right-hand side in Lemma 3.3. but which cannot be handled directly with the help of a Gronwall argument. We start with the following:

Lemma 3.4. We have in $\left[0, \hat{T}_{h}\right)$ and for $\epsilon>0$ :

$$
\left\|\tau-\tau_{h}\right\|^{2} \leq \epsilon\left(\left\|y-y_{h}\right\|^{2}+\left\|\left|x_{u}\right|-\left|x_{h u}\right|\right\|^{2}\right)+C_{\epsilon}\left(h^{2}+\left\|x-x_{h}\right\|^{2}\right) .
$$

Proof. We deduce from (2.3) and (2.10) that

$$
\begin{array}{r}
\int_{0}^{2 \pi}\left(\tau-\tau_{h}, \psi_{h u}\right)=-\int_{0}^{2 \pi}\left(y-I_{h} y, \psi_{h}\right)\left|x_{h u}\right|-\left(y, \psi_{h}\right)\left(\left|x_{u}\right|-\left|x_{h u}\right|\right) \\
\quad-\int_{0}^{2 \pi} I_{h}\left[\left(I_{h} y-y_{h}, \psi_{h}\right)\right]\left|x_{h u}\right|+\frac{1}{6} \sum_{j=1}^{N} h_{j}^{2} \int_{I_{j}}\left(\left(I_{h} y\right)_{u}, \psi_{h u}\right)\left|x_{h u}\right|
\end{array}
$$

for $\psi_{h} \in X_{h}^{n}$. Inserting $\psi_{h}=I_{h} x-x_{h}$ we derive

$$
\begin{aligned}
& \int_{0}^{2 \pi}\left(\tau-\tau_{h}, x_{u}-x_{h u}\right) \\
& \quad \leq \delta\left\|\tau-\tau_{h}\right\|^{2}+C_{\delta} h^{2}+\epsilon\left(\left\|y-y_{h}\right\|^{2}+\left\|\left|x_{u}\right|-\left|x_{h u}\right|\right\|^{2}\right)+C_{\epsilon}\left(h^{2}+\left\|x-x_{h}\right\|^{2}\right) .
\end{aligned}
$$

Using (3.4) we see that

$$
\left(\tau-\tau_{h}, x_{u}-x_{h u}\right)=\left(\tau-\tau_{h},\left|x_{u}\right| \tau-\left|x_{h u}\right| \tau_{h}\right)=\frac{1}{2}\left|\tau-\tau_{h}\right|^{2}\left(\left|x_{u}\right|+\left|x_{h u}\right|\right),
$$

and we complete the proof by choosing $\delta$ small enough.

In what follows it will be convenient to write the finite element scheme as a difference scheme. To do so, we introduce the quantities

$$
x_{j}=x_{h}\left(u_{j}\right), y_{j}=y_{h}\left(u_{j}\right), q_{j}=\left|x_{j}-x_{j-1}\right|, \alpha_{j}=\frac{1}{2}\left(q_{j}+q_{j+1}\right), \tau_{j}=\frac{x_{j}-x_{j-1}}{\left|x_{j}-x_{j-1}\right|}
$$

for $j=1, \ldots, N$ where here and in the following we use $N$-periodic indices, e.g. $x_{N+1}=x_{1}$. We insert $\phi_{h}, \psi_{h}=\varphi_{j} e^{k}(k=1, \ldots, N)$ as test functions into (2.9), (2.10), respectively, and obtain

$$
\begin{aligned}
\alpha_{j} \dot{x}_{j}+\frac{1}{q_{j+1}} P_{j+1}\left(y_{j+1}-y_{j}\right)-\frac{1}{q_{j}} P_{j}\left(y_{j}-y_{j-1}\right) & \\
+\frac{1}{4}\left(\left(\left|y_{j}\right|^{2}+\left|y_{j+1}\right|^{2}\right) \tau_{j+1}-\left(\left|y_{j-1}\right|^{2}+\left|y_{j}\right|^{2}\right) \tau_{j}\right)-\lambda\left(\tau_{j+1}-\tau_{j}\right) & =0, \\
\alpha_{j} y_{j}-\left(\tau_{j+1}-\tau_{j}\right) & =0,
\end{aligned}
$$

where we have used the abbreviation $P_{j}=I-\tau_{j} \otimes \tau_{j}$. Note that (3.4) and (3.16) imply

$$
\left(\tau_{j+1}-\tau_{j}, \tau_{j}\right)=-\frac{1}{2}\left|\tau_{j+1}-\tau_{j}\right|^{2}=-\frac{1}{2} \alpha_{j}^{2}\left|y_{j}\right|^{2}
$$


and hence

$$
\left(\tau_{j+1}, y_{j}\right)=\frac{1}{\alpha_{j}}\left(\tau_{j+1}, \tau_{j+1}-\tau_{j}\right)=\frac{1}{2} \alpha_{j}\left|y_{j}\right|^{2}, \quad\left(\tau_{j}, y_{j}\right)=-\frac{1}{2} \alpha_{j}\left|y_{j}\right|^{2} .
$$

Lemma 3.5. We have for $\epsilon>0$,

$$
\left\|y_{u}-y_{h u}\right\|^{2} \leq \epsilon\left\|x_{t}-x_{h t}\right\|^{2}+C_{\epsilon}\left(h^{2}+\left\|x-x_{h}\right\|^{2}+\left\|y-y_{h}\right\|^{2}+\left\|\left|x_{u}\right|-\left|x_{h u}\right|\right\|^{2}\right) .
$$

Proof. Let us split the difference $y_{u}-y_{h u}$ as follows:

$$
y_{u}-y_{h u}=P_{h}\left(y_{u}-y_{h u}\right)+\left(y_{u}-y_{h u}, \tau_{h}\right) \tau_{h} .
$$

In order to estimate the first term on the right-hand side we note that (2.2), (2.9) as well as Remark 2.1 imply

$$
\begin{aligned}
\int_{0}^{2 \pi} \frac{1}{\left|x_{h u}\right|} & \left(P_{h}\left(y_{u}-y_{h u}\right), \phi_{h u}\right)=\int_{0}^{2 \pi}\left(\frac{1}{\left|x_{h u}\right|} P_{h} y_{u}-\frac{1}{\left|x_{u}\right|} P y_{u}, \phi_{h u}\right) \\
& +\int_{0}^{2 \pi}\left\{\left(x_{t}, \phi_{h}\right)\left|x_{u}\right|-I_{h}\left[\left(x_{h t}, \phi_{h}\right)\right]\left|x_{h u}\right|\right\} \\
& -\frac{1}{2} \int_{0}^{2 \pi}\left\{|y|^{2}\left(\phi_{h u}, \tau\right)-I_{h}\left[\left|y_{h}\right|^{2}\right]\left(\phi_{h u}, \tau_{h}\right)\right\} \\
& -\lambda \int_{0}^{2 \pi}\left\{\left(y, \phi_{h}\right)\left|x_{u}\right|-I_{h}\left[\left(y_{h}, \phi_{h}\right)\right]\left|x_{h u}\right|\right\}
\end{aligned}
$$

for $\phi_{h} \in X_{h}^{n}$. Inserting $\phi_{h}=I_{h} y-y_{h}$ into (3.19) and using similar arguments as above we obtain, in view of (3.3),

$$
\begin{aligned}
& \frac{1}{2 C_{0}}\left\|P_{h}\left(y_{u}-y_{h u}\right)\right\|^{2} \leq \delta\left\|y_{u}-y_{h u}\right\|^{2}+\epsilon\left\|x_{t}-x_{h t}\right\|^{2} \\
& \quad+\left(C_{\delta}+C_{\epsilon}\right)\left(h^{2}+\left\|\tau-\tau_{h}\right\|^{2}+\left\|y-y_{h}\right\|^{2}+\left\|\left|x_{u}\right|-\left|x_{h u}\right|\right\|^{2}\right) .
\end{aligned}
$$

Let us next consider the term $\left(y_{u}-y_{h u}, \tau_{h}\right) \tau_{h}$. We first observe that

$$
\left(y_{u}, \tau\right)=(y, \tau)_{u}-\left(y, \tau_{u}\right)=-\left|x_{u}\right||y|^{2} \quad \text { in }[0,2 \pi] \times[0, T] .
$$

The discrete analogue of this relation follows from (3.17), namely

$$
\left(y_{j}-y_{j-1}, \tau_{j}\right)=-\frac{1}{2} \alpha_{j}\left|y_{j}\right|^{2}-\frac{1}{2} \alpha_{j-1}\left|y_{j-1}\right|^{2}
$$

where we have used the notation introduced above. Combining (3.21) and (3.22) we find in $I_{j}$,

$$
\begin{aligned}
\left|\left(y_{u}-y_{h u}, \tau_{j}\right)\right| \leq & C\left|\tau-\tau_{j}\right|+\left.|| x_{u}|-| x_{h u}\right|_{I_{j}} \mid+C\left(\left|y-y_{j-1}\right|+\left|y-y_{j}\right|\right) \\
& +\frac{C}{h_{j}}\left(\left|q_{j}-q_{j-1}\right|+\left|q_{j+1}-q_{j}\right|\right) .
\end{aligned}
$$

Combining (2.5) with an inverse estimate we find that

$$
\begin{gathered}
\left|q_{j+1}-q_{j}\right| \leq h_{j+1}||\left(I_{h} x\right)_{u}|-| x_{h u}||||_{I_{j+1}}+C h^{2}+\left.h_{j}||\left(I_{h} x\right)_{u}|-| x_{h u}\right|_{I_{j}} \\
\leq C \sqrt{h}\left\|\left|\left(I_{h} x\right)_{u}\right|-\left|x_{h u}\right|\right\|_{L^{2}\left(I_{j} \cup I_{j+1}\right)}+C h^{2},
\end{gathered}
$$


and therefore

$$
\begin{aligned}
& \int_{0}^{2 \pi}\left(y_{u}-y_{h u}, \tau_{h}\right)^{2}=\sum_{j=1}^{N} \int_{I_{j}}\left(y_{u}-y_{h u}, \tau_{j}\right)^{2} \\
& \quad \leq C h^{2}+C \sum_{j=1}^{N}\left\{\int_{I_{j}}\left(\left|\tau-\tau_{h}\right|^{2}+\left|y-y_{h}\right|^{2}\right)+\left.\int_{I_{j-1} \cup I_{j} \cup I_{j+1}}|| x_{u}|-| x_{h u}\right|^{2}\right\} \\
& \quad \leq C\left(h^{2}+\left\|\tau-\tau_{h}\right\|^{2}+\left\|y-y_{h}\right\|^{2}+\left\|\left|x_{u}\right|-\left|x_{h u}\right|\right\|^{2}\right) .
\end{aligned}
$$

This estimate together with (3.18), (3.20) and Lemma 3.4yields the desired bound after choosing $\delta$ sufficiently small.

The most difficult part is to estimate the error in the length element. The basis is an ODE with respect to time for the lengths of the polygonal curve. In order to motivate the structure of this ODE we note that (1.2) implies that $\left(x_{t}, \tau\right)=0$ and hence by (3.6) and the definition of $y$,

$$
\left|x_{u}\right|_{t}=\left(\tau, x_{u t}\right)=\left(\tau, x_{t}\right)_{u}-\left(\tau_{u}, x_{t}\right)=-\left(\tau_{u}, x_{t}\right)=-\left(y, x_{t}\right)\left|x_{u}\right| .
$$

Lemma 3.6. The discrete length element satisfies

$$
\dot{q}_{j}+\frac{1}{2}\left(\left(\dot{x}_{j}, y_{j}\right)+\left(\dot{x}_{j-1}, y_{j-1}\right)\right) q_{j}=R_{j}
$$

with $R_{j}=S_{j}-S_{j-1}$ and

$$
\begin{gathered}
S_{j}=-\frac{1}{4 \alpha_{j}}\left(\left|y_{j+1}-y_{j}\right|^{2}-\left|y_{j}-y_{j-1}\right|^{2}\right)+\frac{1}{16}\left|y_{j}\right|^{4}\left(q_{j+1}-q_{j}\right)-\frac{\lambda}{4}\left|y_{j}\right|^{2}\left(q_{j+1}-q_{j}\right) \\
+\frac{1}{16}\left|y_{j}\right|^{2}\left|y_{j+1}\right|^{2}\left(q_{j+2}+2 q_{j+1}\right)-\frac{1}{16}\left|y_{j}\right|^{2}\left|y_{j-1}\right|^{2}\left(q_{j-1}+2 q_{j}\right)
\end{gathered}
$$

for $j=1, \ldots, N$ with $N$-periodic indexing.

Proof. Obviously,

$$
\dot{q}_{j}=\left(\tau_{j}, \dot{x}_{j}\right)-\left(\tau_{j}, \dot{x}_{j-1}\right) .
$$

Let us calculate the expressions on the right-hand side of this equation. For practical reasons we use the abbreviation

$$
J_{j}=\frac{1}{2}\left(\left|y_{j-1}\right|^{2}+\left|y_{j}\right|^{2}\right) .
$$

From (3.15) and $P_{j} \tau_{j}=0$ we deduce

$$
\begin{aligned}
& \alpha_{j}\left(\tau_{j}, \dot{x}_{j}\right) \\
& \quad=-\frac{1}{q_{j+1}}\left(\tau_{j}, P_{j+1}\left(y_{j+1}-y_{j}\right)\right)+\frac{1}{2}\left(J_{j}-\left(\tau_{j+1}, \tau_{j}\right) J_{j+1}\right)+\lambda\left(\tau_{j+1}-\tau_{j}, \tau_{j}\right) .
\end{aligned}
$$

Next, (3.4) and (3.16) imply that

$$
\begin{aligned}
P_{j+1} \tau_{j} & =\tau_{j}-\left(\tau_{j+1}, \tau_{j}\right) \tau_{j+1}=\tau_{j}-\tau_{j+1}+\frac{1}{2}\left|\tau_{j+1}-\tau_{j}\right|^{2} \tau_{j+1} \\
& =-\alpha_{j} y_{j}+\frac{1}{2} \alpha_{j}^{2}\left|y_{j}\right|^{2} \tau_{j+1},
\end{aligned}
$$


and using again (3.4) we deduce

$$
\begin{gathered}
\alpha_{j}\left(\tau_{j}, \dot{x}_{j}\right)=\frac{\alpha_{j}}{q_{j+1}}\left(y_{j}, y_{j+1}-y_{j}\right)-\frac{\alpha_{j}^{2}}{2 q_{j+1}}\left|y_{j}\right|^{2}\left(\tau_{j+1}, y_{j+1}-y_{j}\right) \\
+\frac{1}{2}\left(J_{j}-J_{j+1}\right)+\frac{1}{4} \alpha_{j}^{2}\left|y_{j}\right|^{2} J_{j+1}-\frac{\lambda}{2} \alpha_{j}^{2}\left|y_{j}\right|^{2} .
\end{gathered}
$$

The elementary relation $\left(y_{j}, y_{j+1}\right)=-\frac{1}{2}\left|y_{j+1}-y_{j}\right|^{2}+\frac{1}{2}\left|y_{j+1}\right|^{2}+\frac{1}{2}\left|y_{j}\right|^{2}$ together with (3.17) finally implies that

$$
\begin{aligned}
\left(\dot{x}_{j}, \tau_{j}\right)= & \frac{1}{2 q_{j+1}}\left(\left|y_{j+1}\right|^{2}-\left|y_{j}\right|^{2}\right)-\frac{1}{2 q_{j+1}}\left|y_{j+1}-y_{j}\right|^{2}+\frac{1}{2 \alpha_{j}}\left(J_{j}-J_{j+1}\right) \\
& +\frac{\alpha_{j}}{4 q_{j+1}}\left|y_{j}\right|^{2}\left(\alpha_{j+1}\left|y_{j+1}\right|^{2}+\alpha_{j}\left|y_{j}\right|^{2}\right)+\frac{1}{4} \alpha_{j}\left|y_{j}\right|^{2} J_{j+1}-\frac{\lambda}{2} \alpha_{j}\left|y_{j}\right|^{2} .
\end{aligned}
$$

Similar calculations lead to the equation

$$
\begin{gathered}
\left(\dot{x}_{j-1}, \tau_{j}\right)=\frac{1}{2 q_{j-1}}\left(\left|y_{j-1}\right|^{2}-\left|y_{j-2}\right|^{2}\right)+\frac{1}{2 q_{j-1}}\left|y_{j-1}-y_{j-2}\right|^{2}+\frac{1}{2 \alpha_{j-1}}\left(J_{j-1}-J_{j}\right) \\
\quad-\frac{\alpha_{j-1}}{4 q_{j-1}}\left|y_{j-1}\right|^{2}\left(\alpha_{j-1}\left|y_{j-1}\right|^{2}+\alpha_{j-2}\left|y_{j-2}\right|^{2}\right) \\
\quad-\frac{1}{4} \alpha_{j-1}\left|y_{j-1}\right|^{2} J_{j-1}+\frac{\lambda}{2} \alpha_{j-1}\left|y_{j-1}\right|^{2} .
\end{gathered}
$$

In order to derive an equation of the form (3.25) we still have to rewrite the euclidean product between $\dot{x}_{j}$ and $y_{j}$. Recalling (3.17) we obtain

$$
P_{j} y_{j}=y_{j}-\left(y_{j}, \tau_{j}\right) \tau_{j}=y_{j}+\frac{1}{2} \alpha_{j}\left|y_{j}\right|^{2} \tau_{j}, \quad P_{j+1} y_{j}=y_{j}-\frac{1}{2} \alpha_{j}\left|y_{j}\right|^{2} \tau_{j+1} .
$$

With these relations we obtain from (3.15)

$$
\begin{gathered}
\alpha_{j}\left(\dot{x}_{j}, y_{j}\right)=\frac{1}{q_{j}}\left(y_{j}+\frac{1}{2} \alpha_{j}\left|y_{j}\right|^{2} \tau_{j}, y_{j}-y_{j-1}\right)-\frac{1}{q_{j+1}}\left(y_{j}-\frac{1}{2} \alpha_{j}\left|y_{j}\right|^{2} \tau_{j+1}, y_{j+1}-y_{j}\right) \\
-\frac{1}{4}\left(\alpha_{j}\left|y_{j}\right|^{2} J_{j}+\alpha_{j}\left|y_{j}\right|^{2} J_{j+1}\right)+\lambda \alpha_{j}\left|y_{j}\right|^{2}
\end{gathered}
$$

and hence, with the help of (3.17),

$$
\begin{aligned}
\left(\dot{x}_{j}, y_{j}\right)= & -\frac{1}{2 \alpha_{j} q_{j+1}}\left(\left|y_{j+1}\right|^{2}-\left|y_{j}\right|^{2}\right)+\frac{1}{2 \alpha_{j} q_{j}}\left(\left|y_{j}\right|^{2}-\left|y_{j-1}\right|^{2}\right) \\
& +\frac{1}{2 \alpha_{j} q_{j+1}}\left|y_{j+1}-y_{j}\right|^{2}+\frac{1}{2 \alpha_{j} q_{j}}\left|y_{j}-y_{j-1}\right|^{2} \\
& -\frac{1}{4 q_{j}}\left|y_{j}\right|^{2}\left(\alpha_{j}\left|y_{j}\right|^{2}+\alpha_{j-1}\left|y_{j-1}\right|^{2}\right) \\
& -\frac{1}{4 q_{j+1}}\left|y_{j}\right|^{2}\left(\alpha_{j+1}\left|y_{j+1}\right|^{2}+\alpha_{j}\left|y_{j}\right|^{2}\right) \\
& -\frac{1}{4}\left|y_{j}\right|^{2}\left(J_{j}+J_{j+1}\right)+\lambda\left|y_{j}\right|^{2}
\end{aligned}
$$


Let us combine (3.28), (3.29) with (3.31) for $j$ and $j-1$. Recalling the definition of $J_{j}$ and sorting terms we obtain after some elementary calculations

$$
\begin{aligned}
\dot{q}_{j} & +\frac{1}{2}\left(\left(\dot{x}_{j}, y_{j}\right)+\left(\dot{x}_{j-1}, y_{j-1}\right)\right) q_{j} \\
= & \left(\tau_{j}, \dot{x}_{j}\right)-\left(\tau_{j}, \dot{x}_{j-1}\right)+\frac{1}{2} q_{j}\left(\dot{x}_{j}, y_{j}\right)+\frac{1}{2} q_{j}\left(\dot{x}_{j-1}, y_{j-1}\right) \\
= & \left(\frac{1}{2 q_{j+1}}-\frac{q_{j}}{4 q_{j+1} \alpha_{j}}\right)\left(\left|y_{j+1}\right|^{2}-\left|y_{j}\right|^{2}\right)+\frac{1}{4 \alpha_{j}}\left(\left|y_{j-1}\right|^{2}-\left|y_{j+1}\right|^{2}\right) \\
& -\frac{1}{4 \alpha_{j-1}}\left(\left|y_{j-2}\right|^{2}-\left|y_{j}\right|^{2}\right)+\left(\frac{q_{j}}{4 q_{j-1} \alpha_{j-1}}-\frac{1}{2 q_{j-1}}\right)\left(\left|y_{j-1}\right|^{2}-\left|y_{j-2}\right|^{2}\right) \\
& +\frac{1}{4 \alpha_{j}}\left(\left|y_{j}\right|^{2}-\left|y_{j-1}\right|^{2}\right)-\frac{1}{4 \alpha_{j-1}}\left(\left|y_{j}\right|^{2}-\left|y_{j-1}\right|^{2}\right) \\
& -\left(\frac{1}{2 q_{j-1}}-\frac{q_{j}}{4 \alpha_{j-1} q_{j-1}}\right)\left|y_{j-1}-y_{j-2}\right|^{2}-\left(\frac{1}{2 q_{j+1}}-\frac{q_{j}}{4 \alpha_{j} q_{j+1}}\right)\left|y_{j+1}-y_{j}\right|^{2} \\
& +\left(\frac{1}{4 \alpha_{j}}+\frac{1}{4 \alpha_{j-1}}\right)\left|y_{j}-y_{j-1}\right|^{2}+\left(\frac{\alpha_{j}}{4 q_{j+1}}-\frac{q_{j}}{8 q_{j+1}}\right)\left(\alpha_{j}\left|y_{j}\right|^{2}+\alpha_{j+1}\left|y_{j+1}\right|^{2}\right)\left|y_{j}\right|^{2} \\
& +\left(\frac{\alpha_{j-1}}{4 q_{j-1}}-\frac{q_{j}}{8 q_{j-1}}\right)\left(\alpha_{j-2}\left|y_{j-2}\right|^{2}+\alpha_{j-1}\left|y_{j-1}\right|^{2}\right)\left|y_{j-1}\right|^{2} \\
& -\frac{1}{8}\left(\left|y_{j-1}\right|^{2}+\left|y_{j}\right|^{2}\right)\left(\alpha_{j-1}\left|y_{j-1}\right|^{2}+\alpha_{j}\left|y_{j}\right|^{2}\right)+\frac{\alpha_{j}}{8}\left|y_{j}\right|^{2}\left(\left|y_{j}\right|^{2}+\left|y_{j+1}\right|^{2}\right) \\
& +\frac{\alpha_{j-1}}{8}\left|y_{j-1}\right|^{2}\left(\left|y_{j-2}\right|^{2}+\left|y_{j-1}\right|^{2}\right)-\frac{q_{j}}{16}\left|y_{j}\right|^{2}\left(\left|y_{j-1}\right|^{2}+2\left|y_{j}\right|^{2}+\left|y_{j+1}\right|^{2}\right) \\
& -\frac{q_{j}}{16}\left|y_{j-1}\right|^{2}\left(\left|y_{j-2}\right|^{2}+2\left|y_{j-1}\right|^{2}+\left|y_{j}\right|^{2}\right)-\frac{\lambda}{2}\left(\alpha_{j-1}\left|y_{j-1}\right|^{2}+\alpha_{j}\left|y_{j}\right|^{2}\right) \\
& +\frac{\lambda}{2} q_{j}\left(\left|y_{j-1}\right|^{2}+\left|y_{j}\right|^{2}\right) .
\end{aligned}
$$

We observe that

$$
\begin{aligned}
\frac{1}{2 q_{j+1}}-\frac{q_{j}}{4 q_{j+1} \alpha_{j}} & =\frac{1}{4 \alpha_{j}}, \quad \frac{q_{j}}{4 q_{j-1} \alpha_{j-1}}-\frac{1}{2 q_{j-1}}=-\frac{1}{4 \alpha_{j-1}}, \\
\frac{\alpha_{j}}{4 q_{j+1}}-\frac{q_{j}}{8 q_{j+1}} & =\frac{\alpha_{j-1}}{4 q_{j-1}}-\frac{q_{j}}{8 q_{j-1}}=\frac{1}{8}
\end{aligned}
$$

and after rearranging and simplifying, we obtain the claim of the lemma.

Lemma 3.7. We have for $t \in\left[0, \hat{T}_{h}\right)$,

$$
\left\|\left(\left|x_{u}\right|-\left|x_{h u}\right|\right)(\cdot, t)\right\|^{2} \leq C h^{2}+C \int_{0}^{t}\left\|x-x_{h}\right\|^{2}+\left\|y-y_{h}\right\|^{2}+\left\|x_{t}-x_{h t}\right\|^{2} d t .
$$

Proof. Let us define $\tilde{x}_{h}=I_{h} x, \tilde{y}_{h}=I_{h} y$ and introduce the quantities

$$
\tilde{x}_{j}=x\left(u_{j}\right), \tilde{y}_{j}=y\left(u_{j}\right), \tilde{q}_{j}=\left|\tilde{x}_{j}-\tilde{x}_{j-1}\right|, \tilde{\alpha}_{j}=\frac{1}{2}\left(\tilde{q}_{j}+\tilde{q}_{j+1}\right), \tilde{\tau}_{j}=\frac{\tilde{x}_{j}-\tilde{x}_{j-1}}{\left|\tilde{x}_{j}-\tilde{x}_{j-1}\right|} .
$$

From (3.24) and Lemma 3.6 we infer that on the grid interval $I_{j}$,

$$
\left(\left|x_{u}\right|-\left|x_{h u}\right|\right)_{t}=-\left(x_{t}, y\right)\left|x_{u}\right|+\frac{1}{2 h_{j}}\left(\left(\dot{x}_{j}, y_{j}\right)+\left(\dot{x}_{j-1}, y_{j-1}\right)\right) q_{j}-\frac{1}{h_{j}} R_{j}
$$


and hence

$$
\begin{aligned}
& \left|\left(\left|x_{u}\right|-\left|x_{h u}\right|\right)(\cdot, t)\right|-\left|\left(x_{u}-x_{h u}\right)(\cdot, 0)\right|-\frac{1}{h_{j}} \int_{0}^{t}\left|R_{j}\right| d t \\
& \leq \quad \frac{1}{2 h_{j}} \int_{0}^{t}\left|\left(\left(\dot{\tilde{x}}_{j}, \tilde{y}_{j}\right)+\left(\dot{\tilde{x}}_{j-1}, \tilde{y}_{j-1}\right)\right) \tilde{q}_{j}-\left(\left(\dot{x}_{j}, y_{j}\right)+\left(\dot{x}_{j-1}, y_{j-1}\right)\right) q_{j}\right| d t \\
& \quad+\frac{1}{h_{j}} \int_{0}^{t}\left|\frac{1}{2}\left(\left(\dot{\tilde{x}}_{j}, \tilde{y}_{j}\right)+\left(\dot{\tilde{x}}_{j-1}, \tilde{y}_{j-1}\right)\right)\right| \tilde{x}_{j}-\tilde{x}_{j-1}\left|-h_{j}\left(x_{t}, y\right)\right| x_{u}|| d t \\
& \leq \quad C \int_{0}^{t}\left|\tilde{y}_{j}-y_{j}\right|+\left|\dot{\tilde{x}}_{j}-\dot{x}_{j}\right|+\left|\tilde{y}_{j-1}-y_{j-1}\right|+\left|\dot{\tilde{x}}_{j-1}-\dot{x}_{j-1}\right| d t \\
& \quad+C \frac{1}{h_{j}} \int_{0}^{t}\left|\tilde{q}_{j}-q_{j}\right| d t+C h_{j} \\
& \leq \quad \frac{C}{\sqrt{h}} \int_{0}^{t}\left\|\tilde{y}_{h}-y_{h}\right\|_{L^{2}\left(I_{j}\right)}+\left\|\tilde{x}_{h t}-x_{h t}\right\|_{L^{2}\left(I_{j}\right)}+\left\|\left|\tilde{x}_{h u}\right|-\left|x_{h u}\right|\right\|_{L^{2}\left(I_{j}\right)} d t+C h \\
& \leq \frac{C}{\sqrt{h}} \int_{0}^{t}\left\|y-y_{h}\right\|_{L^{2}\left(I_{j}\right)}+\left\|x_{t}-x_{h t}\right\|_{L^{2}\left(I_{j}\right)}+\left\|\left|x_{u}\right|-\left|x_{h u}\right|\right\|_{L^{2}\left(I_{j}\right)} d t+C h .
\end{aligned}
$$

Here we have used the assumptions (3.2) and the smoothness of the continuous solution. Next, we estimate the remainder term $R_{j}$. According to Lemma 3.6.

$$
R_{j}=S_{j}-S_{j-1} \quad \text { with } S_{j}=S_{j}\left(q_{j-1}, q_{j}, q_{j+1}, q_{j+2}, y_{j-1}, y_{j}, y_{j+1}\right) .
$$

One easily shows by Taylor expansion that

$$
\left|\tilde{S}_{j}\right|=\left|S_{j}\left(\tilde{q}_{j-1}, \tilde{q}_{j}, \tilde{q}_{j+1}, \tilde{q}_{j+2}, \tilde{y}_{j-1}, \tilde{y}_{j}, \tilde{y}_{j+1}\right)\right| \leq C h^{2} .
$$

We demonstrate this for two typical terms:

$$
\begin{gathered}
\frac{1}{4 \tilde{\alpha}_{j}}|| \tilde{y}_{j}-\left.\tilde{y}_{j-1}\right|^{2}-\left|\tilde{y}_{j+1}-\tilde{y}_{j}\right|^{2}\left|\leq \frac{C}{h}\right| \tilde{y}_{j-1}-2 \tilde{y}_{j}+\tilde{y}_{j+1}|| \tilde{y}_{j+1}-\tilde{y}_{j-1} \mid \leq C h^{2}, \\
\left|\tilde{q}_{j+1}-\tilde{q}_{j}\right|=|| \tilde{x}_{j+1}-\tilde{x}_{j}|-| \tilde{x}_{j}-\tilde{x}_{j-1}|| \leq\left|\tilde{x}_{j+1}-2 \tilde{x}_{j}+\tilde{x}_{j-1}\right| \leq C h^{2} .
\end{gathered}
$$

Altogether we have with $\tilde{R}_{j}=\tilde{S}_{j}-\tilde{S}_{j-1}$ that

$$
\left|R_{j}\right| \leq\left|R_{j}-\tilde{R}_{j}\right|+C h^{2}
$$

and it remains to estimate the difference between $R_{j}$ and $\tilde{R}_{j}$.

We treat the terms in (3.34) separately. The smoothness assumptions on the continuous solution implies the estimate

$$
\left|q_{j}-\tilde{q}_{j}\right| \leq \sqrt{h_{j}}\left\|\left|x_{u}\right|-\left|x_{h u}\right|\right\|_{L^{2}\left(I_{j}\right)}+C h_{j}^{2} .
$$

This inequality will be used often in the remaining estimates. First,

$$
\begin{gathered}
\left|\frac{1}{4 \alpha_{j}}\left(\left|y_{j+1}-y_{j}\right|^{2}-\left|y_{j}-y_{j-1}\right|^{2}\right)-\frac{1}{4 \tilde{\alpha}_{j}}\left(\left|\tilde{y}_{j+1}-\tilde{y}_{j}\right|^{2}-\left|\tilde{y}_{j}-\tilde{y}_{j-1}\right|^{2}\right)\right| \\
\leq C\left(\left|q_{j+1}-\tilde{q}_{j+1}\right|+\left|q_{j}-\tilde{q}_{j}\right|\right) \\
+C h\left(\left.|| y_{h u}\right|^{2}-\left.\left|\tilde{y}_{h u}\right|^{2}\right|_{I_{j+1}}+\left.|| y_{h u}\right|^{2}-\left.\left|\tilde{y}_{h u}\right|^{2}\right|_{I_{j}}\right) \\
\leq C \sqrt{h}\left\|\left|x_{u}\right|-\left|x_{h u}\right|\right\|_{L^{2}\left(I_{j} \cup I_{j+1}\right)}+C h^{2} \\
+C \sqrt{h}\left\|\tilde{y}_{h u}-y_{h u}\right\|_{L^{2}\left(I_{j} \cup I_{j+1}\right)}\left(1+\left\|y_{h u}\right\|_{L^{\infty}}\right) .
\end{gathered}
$$


Also,

$$
\begin{aligned}
& \left.\left|\frac{1}{16}\right| y_{j}\right|^{2}\left|y_{j+1}\right|^{2}\left(q_{j+2}+2 q_{j+1}\right)-\frac{1}{16}\left|\tilde{y}_{j}\right|^{2}\left|\tilde{y}_{j+1}\right|^{2}\left(\tilde{q}_{j+2}+2 \tilde{q}_{j+1}\right) \mid \\
& \leq C h\left\|\tilde{y}_{h}-y_{h}\right\|_{L^{\infty}\left(I_{j+1}\right)}+C\left(\left|q_{j+2}-\tilde{q}_{j+2}\right|+\left|q_{j+1}-\tilde{q}_{j+1}\right|\right) \\
& \leq C \sqrt{h}\left\|\tilde{y}_{h}-y_{h}\right\|_{L^{2}\left(I_{j+1}\right)}+C \sqrt{h}\left\|\left|x_{u}\right|-\left|x_{h u}\right|\right\|_{L^{2}\left(I_{j+1} \cup I_{j+2}\right)}+C h^{2} .
\end{aligned}
$$

Treating the remaining terms in $S_{j}-\tilde{S}_{j}$ in a similar way as above we finally obtain

$$
\begin{aligned}
\left|R_{j}\right| \leq & C h^{2}+C \sqrt{h}\left(\left\|\left|x_{u}\right|-\left|x_{h u}\right|\right\|_{L^{2}\left(I_{(j)}\right)}+\left\|y-y_{h}\right\|_{L^{2}\left(I_{(j)}\right)}\right) \\
& +C \sqrt{h}\left(\left\|y_{u}-y_{h u}\right\|_{L^{2}\left(I_{(j)}\right)}+h\left\|y_{u u}\right\|_{L^{2}\left(I_{(j)}\right)}\right)\left(1+\left\|y_{h u}\right\|_{L^{\infty}}\right)
\end{aligned}
$$

where we have set $I_{(j)}=I_{j-2} \cup I_{j-1} \cup I_{j} \cup I_{j+1} \cup I_{j+2}$. Combining the inequalities (3.33) and (3.37) we arrive at

$$
\begin{aligned}
& \left|\left(\left|x_{u}\right|-\left|x_{h u}\right|\right)(\cdot, t)\right|_{I_{j}} \leq\left|x_{0 u}-x_{h 0 u}\right|_{I_{j}}+C h \\
& \quad+\frac{C}{\sqrt{h}} \int_{0}^{t}\left\|y-y_{h}\right\|_{L^{2}\left(I_{(j)}\right)}+\left\|x_{t}-x_{h t}\right\|_{L^{2}\left(I_{(j)}\right)}+\left\|\left|x_{u}\right|-\left|x_{h u}\right|\right\|_{L^{2}\left(I_{(j)}\right)} d t \\
& \quad+\frac{C}{\sqrt{h}}\left(\int_{0}^{t}\left\|y_{u}-y_{h u}\right\|_{L^{2}\left(I_{(j)}\right)}^{2}+h^{2}\left\|y_{u u}\right\|_{L^{2}\left(I_{(j)}\right)}^{2} d t\right)^{\frac{1}{2}}\left(1+\left(\int_{0}^{t}\left\|y_{h u}\right\|_{L^{\infty}}^{2} d t\right)^{\frac{1}{2}}\right) .
\end{aligned}
$$

We square this result, integrate over $I_{j}$ and then sum from $j=1, \ldots, N$. Recalling that $x_{h 0}=I_{h} x_{0}$ and (3.3) we obtain

$$
\left\|\left(\left|x_{u}\right|-\left|x_{h u}\right|\right)(\cdot, t)\right\|^{2} \leq C h^{2}+C \int_{0}^{t}\left\|y-y_{h}\right\|_{H^{1}}^{2}+\left\|x_{t}-x_{h t}\right\|^{2}+\left\|\left|x_{u}\right|-\left|x_{h u}\right|\right\|^{2} d t .
$$

Lemma 3.5 with $\epsilon=1$ together with a Gronwall argument completes the proof.

We are now in position to complete the error analysis. Lemma 3.3 implies

$$
\frac{c_{0}}{16} \int_{0}^{t}\left\|x_{t}-x_{h t}\right\|^{2} d t+\zeta(t) \leq C h^{2}+C \int_{0}^{t}\left\|y-y_{h}\right\|_{H^{1}}^{2}+\left\|\tau-\tau_{h}\right\|^{2}+\left\|\left|x_{u}\right|-\left|x_{h u}\right|\right\|^{2} d t
$$

since $\zeta(0) \leq C h^{2}$ by Lemma 2.2, Recalling (2.6) and (2.5) it is straightforward to see that

$$
\zeta(t) \geq \frac{c_{0}}{8}\left\|\left(y-y_{h}\right)(\cdot, t)\right\|^{2}-\delta\left\|\left(\left|x_{u}\right|-\left|x_{h u}\right|\right)(\cdot, t)\right\|^{2}-C_{\delta}\left(h^{2}+\left\|\left(\tau-\tau_{h}\right)(\cdot, t)\right\|^{2}\right) .
$$

Hence, in view of Lemma 3.4

$$
\begin{aligned}
& \frac{c_{0}}{16} \int_{0}^{t}\left\|x_{t}-x_{h t}\right\|^{2} d t+\frac{c_{0}}{8}\left\|\left(y-y_{h}\right)(\cdot, t)\right\|^{2} \leq \delta\left\|\left(\left|x_{u}\right|-\left|x_{h u}\right|\right)(\cdot, t)\right\|^{2} \\
& +C_{\delta}\left(h^{2}+\left\|\left(\tau-\tau_{h}\right)(\cdot, t)\right\|^{2}\right)+C \int_{0}^{t}\left\|y-y_{h}\right\|_{H^{1}}^{2}+\left\|\tau-\tau_{h}\right\|^{2}+\left\|\left|x_{u}\right|-\left|x_{h u}\right|\right\|^{2} d t \\
& \leq \quad\left(\delta+\epsilon C_{\delta}\right)\left\|\left(\left|x_{u}\right|-\left|x_{h u}\right|\right)(\cdot, t)\right\|^{2}+\epsilon C_{\delta}\left\|\left(y-y_{h}\right)(\cdot, t)\right\|^{2}+C_{\delta} C_{\epsilon}\left\|\left(x-x_{h}\right)(\cdot, t)\right\|^{2} \\
& \quad+C_{\delta}\left(1+C_{\epsilon}\right) h^{2}+C \int_{0}^{t}\left\|y-y_{h}\right\|_{H^{1}}^{2}+\left\|\tau-\tau_{h}\right\|^{2}+\left\|\left|x_{u}\right|-\left|x_{h u}\right|\right\|^{2} d t .
\end{aligned}
$$


Using Lemma 3.7 and Lemma 3.5 we infer after choosing first $\delta$ and then $\epsilon$ sufficiently small that

$$
\begin{aligned}
& \frac{c_{0}}{32} \int_{0}^{t}\left\|x_{t}-x_{h t}\right\|^{2} d t+\frac{c_{0}}{16}\left\|\left(y-y_{h}\right)(\cdot, t)\right\|^{2} \leq C\left(h^{2}+\left\|\left(x-x_{h}\right)(\cdot, t)\right\|^{2}\right) \\
& +C \int_{0}^{t}\left\|x-x_{h}\right\|^{2}+\left\|y-y_{h}\right\|^{2}+\left\|\tau-\tau_{h}\right\|^{2}+\left\|\left|x_{u}\right|-\left|x_{h u}\right|\right\|^{2} d t .
\end{aligned}
$$

Since

$$
\left\|\left(x-x_{h}\right)(\cdot, t)\right\|^{2}=\left\|\left(x-x_{h}\right)(\cdot, 0)\right\|^{2}+2 \int_{0}^{t} \int_{0}^{2 \pi}\left(x_{t}-x_{h t}\right)\left(x-x_{h}\right)
$$

we infer

$$
\left\|\left(x-x_{h}\right)(\cdot, t)\right\|^{2} \leq C h^{2}+\epsilon \int_{0}^{t}\left\|x_{t}-x_{h t}\right\|^{2} d t+C_{\epsilon} \int_{0}^{t}\left\|x-x_{h}\right\|^{2} d t, \quad \epsilon>0 .
$$

Combining this estimate with (3.38) and using again Lemma 3.4 and Lemma 3.7 we deduce that the function

$\rho(t):=\left\|\left(x-x_{h}\right)(\cdot, t)\right\|^{2}+\left\|\left(y-y_{h}\right)(\cdot, t)\right\|^{2}+\left\|\left(\left|x_{u}\right|-\left|x_{h u}\right|\right)(\cdot, t)\right\|^{2}+\int_{0}^{t}\left\|x_{t}-x_{h t}\right\|^{2} d t$ satisfies

$$
\rho(t) \leq C h^{2}+C \int_{0}^{t} \rho(s) d t, \quad 0 \leq t<\hat{T}_{h} .
$$

Gronwall's lemma implies that $\rho(t) \leq C h^{2}$ for $0 \leq t \leq \hat{T}_{h}$ and hence

$$
\begin{aligned}
\sup _{0 \leq t \leq \hat{T}_{h}}\left(\left\|\left(x-x_{h}\right)(\cdot, t)\right\|_{H^{1}}^{2}+\left\|\left(y-y_{h}\right)(\cdot, t)\right\|^{2}\right) & \\
& +\int_{0}^{\hat{T}_{h}}\left\|y_{u}-y_{h u}\right\|^{2}+\left\|x_{t}-x_{h t}\right\|^{2} d t \leq C h^{2} .
\end{aligned}
$$

Here we used the relation $x_{u}-x_{h u}=\left|x_{u}\right|\left(\tau-\tau_{h}\right)+\left(\left|x_{u}\right|-\left|x_{h u}\right|\right) \tau_{h}$ and note that the constant $C$ only depends on $T, c_{0}, C_{0}$ and norms of the continuous solution.

We can now prove that $\hat{T}_{h}=T$. If not, we would have $\hat{T}_{h}<T$; the smoothness of the solution along with (3.39) and an inverse estimate would then imply that

$$
\left\|\left(x_{u}-x_{h u}\right)(\cdot, t)\right\|_{L^{\infty}},\left\|\left(y-y_{h}\right)(\cdot, t)\right\|_{L^{\infty}} \leq C \sqrt{h}, \quad \int_{0}^{\hat{T}_{h}}\left\|y_{u}-y_{h u}\right\|_{L^{\infty}}^{2} d t \leq C h,
$$

which combined with (3.1) would give

$$
\frac{3}{4} c_{0} \leq\left|x_{h u}\right| \leq \frac{3}{2} C_{0}, \quad\left|y_{h}\right| \leq \frac{3}{2} C_{0} \text { on }[0,2 \pi] \times\left[0, \hat{T}_{h}\right], \quad \int_{0}^{\hat{T}_{h}}\left\|y_{h u}\right\|_{L^{\infty}}^{2} d t \leq \frac{3}{2} C_{0},
$$

provided that $h \leq h_{0}$ and $h_{0}$ is sufficiently small. However, we could then extend the discrete solution to an interval $\left[0, \hat{T}_{h}+\delta\right]$ for some $\delta>0$ with

$$
\frac{1}{2} c_{0} \leq\left|x_{h u}\right| \leq 2 C_{0}, \quad\left|y_{h}\right| \leq 2 C_{0} \text { on }[0,2 \pi] \times\left[0, \hat{T}_{h}+\delta\right], \quad \int_{0}^{\hat{T}_{h}+\delta}\left\|y_{h u}\right\|_{L^{\infty}}^{2} d t \leq 2 C_{0}
$$

contradicting the definition of $\hat{T}_{h}$. Therefore $\hat{T}_{h}=T$ and (3.39) yields the desired error estimates in Theorem 2.3 . 


\section{IMPLEMENTATION AND NUMERICAL RESULTS}

The spatially discrete elastic flow (2.9), 2.10), 2.11) can be written as a system of ODEs for the unknown vector-valued function $x=\left(x_{1}, \ldots, x_{N}\right)$ with $x_{j}=$ $\left(x_{j, 1}, \ldots, x_{j, n}\right)$.

$$
\begin{gathered}
\frac{1}{2}\left(q_{j}+q_{j+1}\right) \dot{x}_{j}+\frac{1}{q_{j+1}} P_{j+1}\left(y_{j+1}-y_{j}\right)-\frac{1}{q_{j}} P_{j}\left(y_{j}-y_{j-1}\right) \\
+\frac{1}{4}\left(\left(\left|y_{j}\right|^{2}+\left|y_{j+1}\right|^{2}\right) \frac{1}{q_{j+1}}\left(x_{j+1}-x_{j}\right)-\left(\left|y_{j-1}\right|^{2}+\left|y_{j}\right|^{2}\right) \frac{1}{q_{j}}\left(x_{j}-x_{j-1}\right)\right) \\
-\lambda\left(\frac{1}{q_{j+1}} x_{j+1}-\left(\frac{1}{q_{j+1}}+\frac{1}{q_{j}}\right) x_{j}+\frac{1}{q_{j}} x_{j-1}\right)=0, \\
\frac{1}{2}\left(q_{j}+q_{j+1}\right) y_{j}-\left(\frac{1}{q_{j+1}} x_{j+1}-\left(\frac{1}{q_{j+1}}+\frac{1}{q_{j}}\right) x_{j}+\frac{1}{q_{j}} x_{j-1}\right)=0
\end{gathered}
$$

for $j=1, \ldots, N$ periodically in $N$. Here we again have used the abbreviations

$$
q_{j}=\left|x_{j}-x_{j-1}\right|, \quad \tau_{j}=\frac{1}{q_{j}}\left(x_{j}-x_{j-1}\right), \quad P_{j}=I-\tau_{j} \otimes \tau_{j} .
$$

The initial value is given by $x_{j}(0)=x_{0}\left(u_{j}\right)$. Note that in this ODE system the grid sizes $h_{j}$ in the parameter interval do not appear. In this sense our algorithm is "intrinsic".

For the implementation we used the following semi-implicit time discretization of (4.1), (4.2). We adopt the generic notation

$$
z^{m}=z(m \tau), \quad m=0, \ldots, m_{T}
$$

with $m_{T} \tau=T$ for the evaluation of the function $z$ on the $m$-th time level.

Algorithm 4.1 (Fully discrete elastic flow). Let $x_{j}^{0}=x_{0}\left(u_{j}\right)$. For the steps $m=$ $0, \ldots, m_{T}-1$ compute

$$
q_{j}^{m}=\left|x_{j}^{m}-x_{j-1}^{m}\right|, \quad \tau_{j}^{m}=\frac{x_{j}^{m}-x_{j-1}^{m}}{q_{j}^{m}}, \quad j=1, \ldots, N
$$

and solve the linear system of equations

$$
\begin{gathered}
\frac{1}{2 \tau}\left(q_{j}^{m}+q_{j+1}^{m}\right) x_{j}^{m+1}+\frac{1}{q_{j+1}^{m}} P_{j+1}^{m}\left(y_{j+1}^{m+1}-y_{j}^{m+1}\right)-\frac{1}{q_{j}^{m}} P_{j}^{m}\left(y_{j}^{m+1}-y_{j-1}^{m+1}\right) \\
+\frac{1}{4}\left(\left(\left|y_{j}^{m}\right|^{2}+\left|y_{j+1}^{m}\right|^{2}\right) \frac{1}{q_{j+1}^{m}}\left(x_{j+1}^{m+1}-x_{j}^{m+1}\right)-\left(\left|y_{j-1}^{m}\right|^{2}+\left|y_{j}^{m}\right|^{2}\right) \frac{1}{q_{j}^{m}}\left(x_{j}^{m+1}-x_{j-1}^{m+1}\right)\right) \\
-\lambda\left(\frac{1}{q_{j+1}^{m}} x_{j+1}^{m+1}-\left(\frac{1}{q_{j+1}^{m}}+\frac{1}{q_{j}^{m}}\right) x_{j}^{m+1}+\frac{1}{q_{j}^{m}} x_{j-1}^{m+1}\right)=\frac{1}{2 \tau}\left(q_{j}^{m}+q_{j+1}^{m}\right) x_{j}^{m}, \\
\frac{1}{2}\left(q_{j}^{m}+q_{j+1}^{m}\right) y_{j}^{m+1}-\left(\frac{1}{q_{j+1}^{m}} x_{j+1}^{m+1}-\left(\frac{1}{q_{j+1}^{m}}+\frac{1}{q_{j}^{m}}\right) x_{j}^{m+1}+\frac{1}{q_{j}^{m}} x_{j-1}^{m+1}\right)=0
\end{gathered}
$$

for $j=1, \ldots, N$ periodically with respect to $N$. 
Let us write the linear algebra form of this algorithm in order to show its simplicity. We define the matrices

$$
\begin{aligned}
M= & \operatorname{diag}\left(\frac{1}{2}\left(q_{j}^{m}+q_{j+1}^{m}\right)\right) \\
S= & \operatorname{tridiag}\left(-\frac{1}{q_{j}^{m}}, \frac{1}{q_{j}^{m}}+\frac{1}{q_{j+1}^{m}},-\frac{1}{q_{j+1}^{m}}\right) \\
S_{k l}= & \operatorname{tridiag}\left(-\frac{1}{q_{j}^{m}}\left(\delta_{k l}-\tau_{j, k}^{m} \tau_{j, l}^{m}\right)\right. \\
& \left.\frac{1}{q_{j}^{m}}\left(\delta_{k l}-\tau_{j, k}^{m} \tau_{j, l}^{m}\right)+\frac{1}{q_{j+1}^{m}}\left(\delta_{k l}-\tau_{j+1, k}^{m} \tau_{j+1, l}^{m}\right),-\frac{1}{q_{j+1}^{m}}\left(\delta_{k l}-\tau_{j+1, k}^{m} \tau_{j+1, l}^{m}\right)\right) \\
R= & \frac{1}{4} \operatorname{tridiag}\left(\frac{1}{q_{j}^{m}}\left(\left|y_{j-1}^{m}\right|^{2}+\left|y_{j}^{m}\right|^{2}\right),\right. \\
& \left.-\frac{1}{q_{j}^{m}}\left(\left|y_{j-1}^{m}\right|^{2}+\left|y_{j}^{m}\right|^{2}\right)-\frac{1}{q_{j+1}^{m}}\left(\left|y_{j}^{m}\right|^{2}+\left|y_{j+1}^{m}\right|^{2}\right), \frac{1}{q_{j+1}^{m}}\left(\left|y_{j}^{m}\right|^{2}+\left|y_{j+1}^{m}\right|^{2}\right)\right)
\end{aligned}
$$

and omit the notation for the time dependence. The meaning of diag is obvious, $M$ is a diagonal matrix. tridiag denotes a tridiagonal matrix except for two entries in the last column of the first row and in the first column of the last row, which are due to the periodicity. If we now denote by

$$
\mathbf{x}_{k}=\left(x_{1, k}, \ldots, x_{N, k}\right), \quad \mathbf{y}_{k}=\left(y_{1, k}, \ldots, y_{N, k}\right),
$$

the coefficient vectors of the $k$-th component, then the system in Algorithm 4.1 can be written as

$$
\begin{aligned}
& \frac{1}{\tau} M \mathbf{x}_{k}^{m+1}-\sum_{l=1}^{n} S_{k l} \mathbf{y}_{l}^{m+1}+R \mathbf{x}_{k}^{m+1}+\lambda S \mathbf{x}_{k}^{m+1}=\frac{1}{\tau} M \mathbf{x}_{k}^{m}, \\
& M \mathbf{y}_{k}^{m+1}+S \mathbf{x}_{k}^{m+1}=0
\end{aligned}
$$

for $k=1, \ldots, n$.

If we eliminate $\mathbf{y}$ from the first equation by using the second equation, then we finally arrive at

$$
\frac{1}{\tau} M \mathbf{x}_{k}^{m+1}+\sum_{l=1}^{n} S_{k l} M^{-1} S \mathbf{x}_{l}^{m+1}+R \mathbf{x}_{k}^{m+1}+\lambda S \mathbf{x}_{k}^{m+1}=\frac{1}{\tau} M \mathbf{x}_{k}^{m},
$$

$(k=1, \ldots, n)$. We solve this linear system of equations with the BICG-method. It should be mentioned that for this system also the CG-method converged.

There is no stability estimate for the fully discrete scheme yet. Experimentally we observed that a time step restriction of the form $\tau \leq \epsilon h^{2}$ was sufficient for stability of the fully discrete scheme. A better choice is, and this is what we used for long time computations,

$$
0<\tau \leq \epsilon \inf _{j=1, \ldots, N}\left|x_{j}-x_{j-1}\right|^{s} .
$$

Here we typically have chosen $\epsilon=0.1$ and $s=2$. In computations with large deformations of the curve which appear in short time we have chosen $s=3$. Note that condition (4.4) makes the time step size time dependent. 
Example 4.1. We start with a test for convergence. For this we use an exact solution of the problem for $\lambda=0$. Following [5] we use

$$
\begin{aligned}
& x(u, t)=(1+2 t)^{\frac{1}{4}}(\cos (u+0.1 \sin u), \sin (u+0.1 \sin u)), \\
& y(u, t)=-(1+2 t)^{-\frac{1}{4}}(\cos (u+0.1 \sin u), \sin (u+0.1 \sin u))
\end{aligned}
$$

for $u \in[0,2 \pi], t \in[0,1]$ and $n=2$. We computed the discrete solution with Algorithm 4.1 and with a time step size $\tau=0.1 h^{2}$. Then we computed the following errors. For generic continuous and discrete functions $z$ and $z_{h}$ we use the notation

$$
\begin{aligned}
& E(\infty, 2, z)=\sup _{1 \leq m \leq m_{T}}\left\|z(\cdot, m \tau)-z_{h}^{m}\right\|, \\
& E(2,2, z)=\left(\tau \sum_{m=1}^{m_{T}}\left\|z(\cdot, m \tau)-z_{h}^{m}\right\|^{2}\right)^{\frac{1}{2}} .
\end{aligned}
$$

We computed the errors $E(\infty, 2, x), E(\infty, 2, y), E\left(2,2, x_{u}\right), E\left(2,2, y_{u}\right)$. For this we used integration formulas which are exact in $\mathbb{P}_{3}$. In addition we computed the errors in the following expression:

$$
E(\infty, \infty, z)=\sup _{1 \leq m \leq m_{T}} \sup _{0 \leq j \leq N}\left|z\left(\left(j+\frac{1}{2}\right) h, m \tau\right)-\frac{1}{2}\left(z_{h}^{m}(j h)+z_{h}^{m}((j+1) h)\right)\right|
$$

for $z=x$ and $z=y$. The results for the position vector $x$ are shown in Table 1 and for the curvature vector $y$ in Table 2, They confirm our theoretical results for the norms which involve gradients and indicate that quadratic convergence can be expected for the $L^{2}$ and $L^{\infty}$ norms. We add Table 3 which shows errors and experimental orders of convergence for $E\left(\infty, 2,\left|x_{u}\right|\right)$ and $E\left(2,2, x_{t}\right)$. The numbers in the tables are not rounded but just cut off after four digits.

TABLE 1. Errors and orders of convergence for the position vector $x$ in Example 4.1 .

\begin{tabular}{|l|ll|ll|ll|}
\hline$h$ & $E(\infty, 2, x)$ & eoc & $E\left(2,2, x_{u}\right)$ & eоc & $E(\infty, \infty, x)$ & eoc \\
\hline 0.6283 & 0.01500 & - & 0.9309 & - & 0.06877 & - \\
\hline 0.3141 & 0.003320 & 2.175 & 0.4720 & 0.979 & 0.01789 & 1.942 \\
\hline 0.1570 & 0.0008437 & 1.976 & 0.2366 & 0.996 & 0.004517 & 1.986 \\
\hline 0.07853 & 0.0002122 & 1.990 & 0.1184 & 0.999 & 0.001132 & 1.996 \\
\hline 0.03926 & 0.00005311 & 1.998 & 0.05921 & 0.999 & 0.0002831 & 1.999 \\
\hline
\end{tabular}


TABLE 2. Errors and orders of convergence for the curvature vector $y$ in Example 4.1 .

\begin{tabular}{|l|ll|ll|ll|}
\hline$h$ & $E(\infty, 2, y)$ & $e o c$ & $E\left(2,2, y_{u}\right)$ & $e o c$ & $E(\infty, \infty, y)$ & $e o c$ \\
\hline 0.6283 & 0.01876 & - & 0.6691 & - & 0.05365 & - \\
\hline 0.3141 & 0.004371 & 2.102 & 0.3413 & 0.971 & 0.01454 & 1.883 \\
\hline 0.1570 & 0.001091 & 2.001 & 0.1712 & 0.995 & 0.003708 & 1.971 \\
\hline 0.07853 & 0.0002733 & 1.998 & 0.08566 & 0.999 & 0.0009314 & 1.993 \\
\hline 0.03926 & 0.00006842 & 1.997 & 0.04283 & 0.999 & 0.0002331 & 1.998 \\
\hline
\end{tabular}

TABLE 3. Errors and orders of convergence for the test Example 4.1.

\begin{tabular}{|l|ll|ll|}
\hline$h$ & $E\left(\infty, 2,\left|x_{u}\right|\right)$ & $e o c$ & $E\left(2,2, x_{t}\right)$ & $e o c$ \\
\hline 0.6283 & 0.08550 & - & 0.01372 & - \\
\hline 0.3141 & 0.03893 & 1.134 & 0.003371 & 2.025 \\
\hline 0.1570 & 0.01863 & 1.062 & 0.0008610 & 1.969 \\
\hline 0.07853 & 0.009201 & 1.018 & 0.0002166 & 1.990 \\
\hline 0.03926 & 0.004585 & 1.004 & 0.00005424 & 1.997 \\
\hline
\end{tabular}

Example 4.2. Next we compute the evolution of an ellipse under the elastic flow. Here we have chosen $x_{0}(u)=(\cos (u), 4 \sin (u), 0)$ for $u \in[0,2 \pi]$ as initial parametrization. We have chosen $x_{h 0, j}=x_{0}((j-1) h)$ and the computational data were $n=3, N=100, h=0.06283, \tau=0.0001240$ and $\lambda=0.025$. In Figure 1 we show the evolving curve. The grid does not degenerate according to our theoretical results. But the difference in size of the faces of the polygon becomes quite large. Note that this is due to the fact that we solve the full PDE (2.2), (2.3) for the position vector $x$. Since the elastic flow of curves has purely normal direction and since we approximate this flow, the discrete solution also has this property approximately. Thus the nodes at regions of high curvature of the initial ellipse have to become close in the end. Since we do not impose tangential motion onto the system this effect has to appear. Otherwise we would not solve the correct problem.

For practical purposes one can introduce tangential motion into the scheme which keeps the grid pleasant. The easiest possibility to do this is to reparametrize the curve after some time steps according to arc length. Another possibility is presented in [1]. We did not use any of these approaches for our computations since we wanted to treat the pure elastic flow problem.

Let us mention that the scheme in [5] is quite similar to our scheme except for integration by parts in the continuous variational equation. That scheme suffered from undesired tangential motions which do not appear in our scheme. Our scheme has the property that it respects the variational structure of the elastic flow 

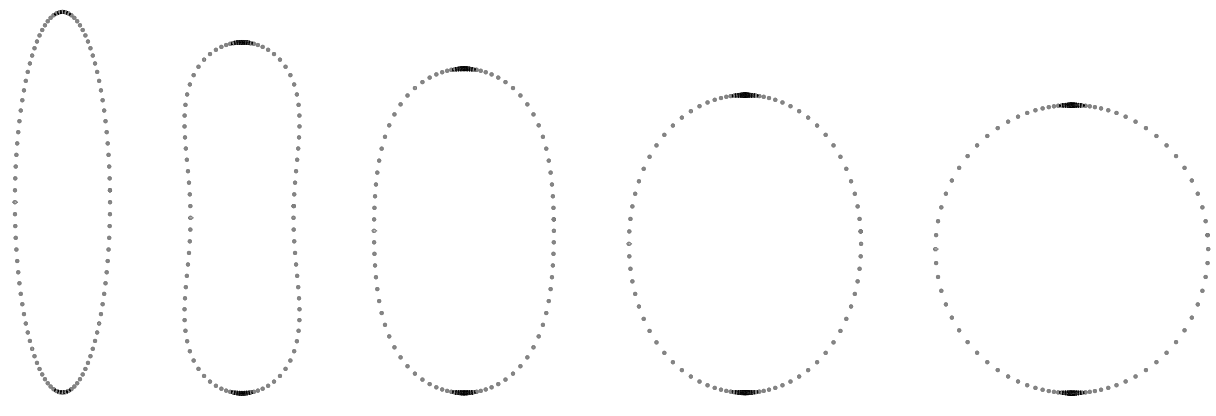

$t=0.0$

$t=1.24$

$$
t=6.2
$$

$t=12.4$

$t=24.8$

Figure 1. Evolution of an ellipse under elastic flow and distribution of the nodes (Example 4.2).

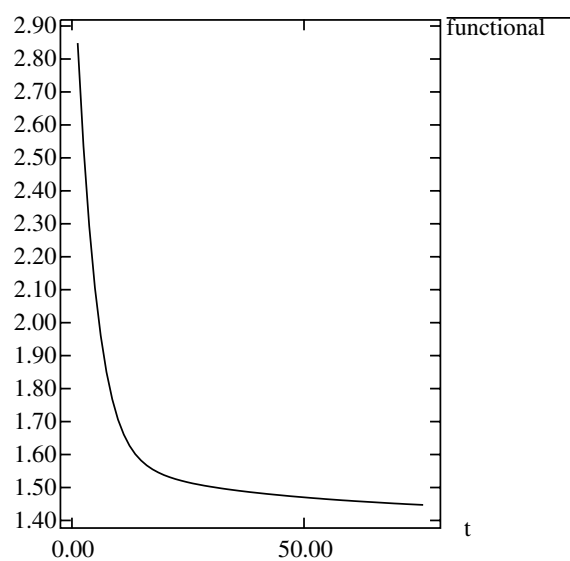

sigma

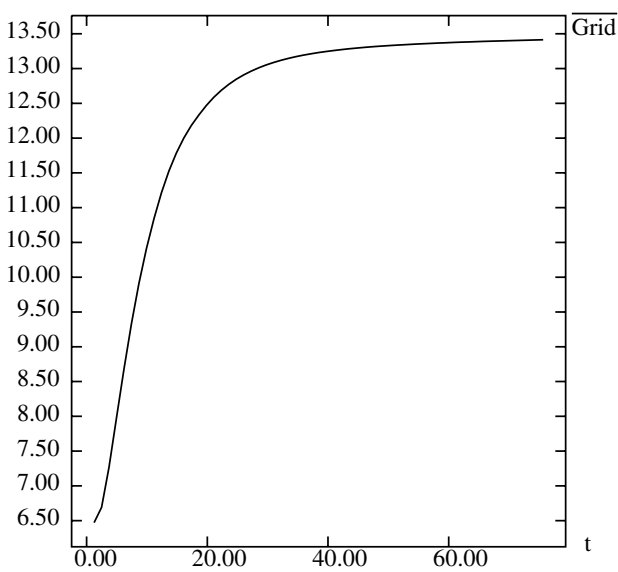

Figure 2. Evolution of the functional $E_{\lambda}$ (left) and evolution of the grid parameter $\sigma$ (right) for Example 4.2.

problem also in the discrete setting; cf. Remark 2.1. However, at present it is unclear whether the energy also decreases for solutions of the fully discrete scheme, Algorithm 4.1

In Figure 2 we present the value of the functional $E_{\lambda}$ together with a graph which describes the behaviour of the grid by showing the evolution of the parameter

$$
\sigma=\frac{\sup _{j=1, \ldots, N}\left|x_{j}-x_{j-1}\right|}{\inf _{j=1, \ldots, N}\left|x_{j}-x_{j-1}\right|}
$$

Example 4.3. We finally compute the evolution under elastic flow for a curve which for $\delta=0$ is a hypocycloid. The parametrization of the initial curve is given by

$$
x_{0}(u)=\left(-\frac{5}{2} \cos (u)+4 \cos (5 u),-\frac{5}{2} \sin (u)+4 \sin (5 u), \delta \sin (3 u)\right) .
$$


It is well known [6] that multiple coverings of a circle are stable stationary solutions for codimension one, i.e. for $n=2$. This is not true for higher codimension $(n \geq 3)$. Obviously for $n=2$ the initial curve evolves to a five-fold covering of a circle (Figure 3). Here the computational parameters are $\lambda=0.025, N=200$ and the time step was chosen as in (4.4) with $\epsilon=0.1$ and $s=2$.

It is worth noting that our scheme keeps the initial curve planar even if we compute in three dimensions, i.e. $n=3$. There are no round off errors introduced into the third component of the solution of the elastic flow.

If we start with an initial curve which is slightly perturbed in a vertical direction, we have chosen $\delta=0.1$, then for "small" times up to about $t=1000.0$ the evolution is quite similar to the two-dimensional case. But then the curve begins to unfold in a complicated manner and evolves to a single circle. This is shown in Figure 6.

The computational parameters are $\lambda=0.025, N=200$ and the time step was chosen as in (4.4) with $\epsilon=0.1$ and $s=3$.
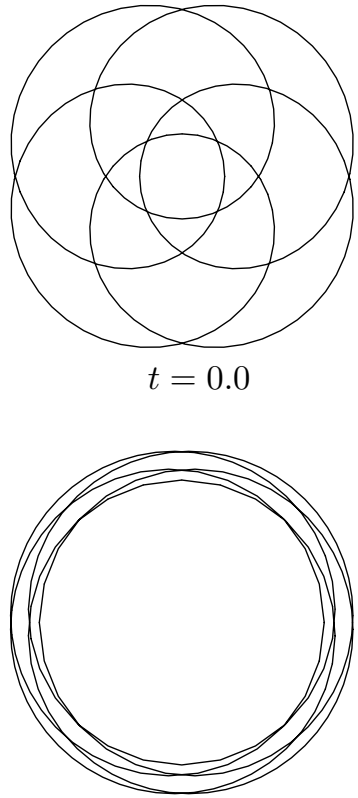

$t=4930.5$

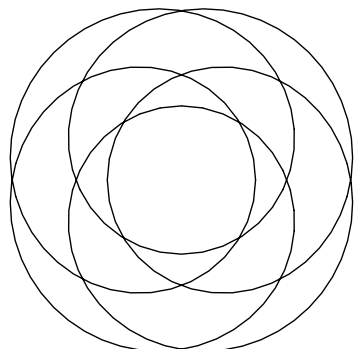

$t=690.1$

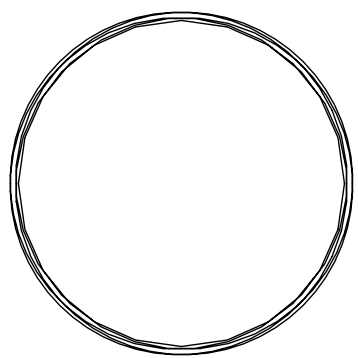

$t=7889.5$

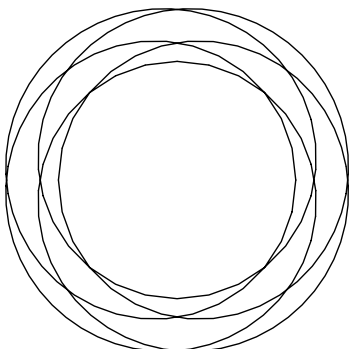

$t=3011.9$

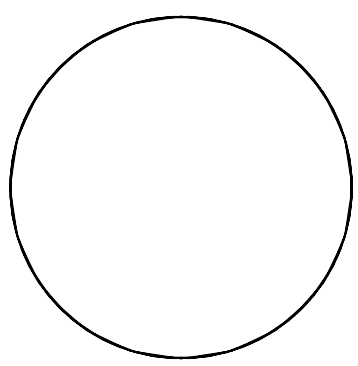

$t=10441.2$

Figure 3. Example 4.3 for $\delta=0$ : evolution of a planar hypocycloid towards a five-fold covering of a circle. The curves are graphically rescaled to have similar size.

In Figure 4 we plot the value of $E_{\lambda}$ against time for planar and perturbed hypocyloid. Finally, Figure 5 shows the evolution of the grid parameter $\sigma$ in both cases. 
E

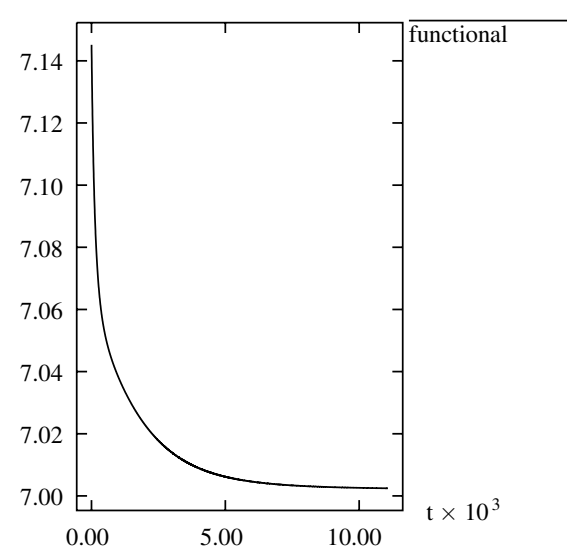

E

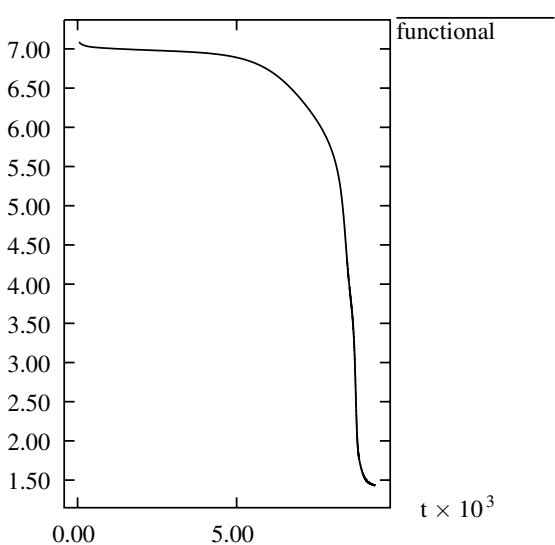

Figure 4. Value of the functional during the evolution for Example 4.3 for $\delta=0$ (left) and $\delta=0.1$ (right).

sigma

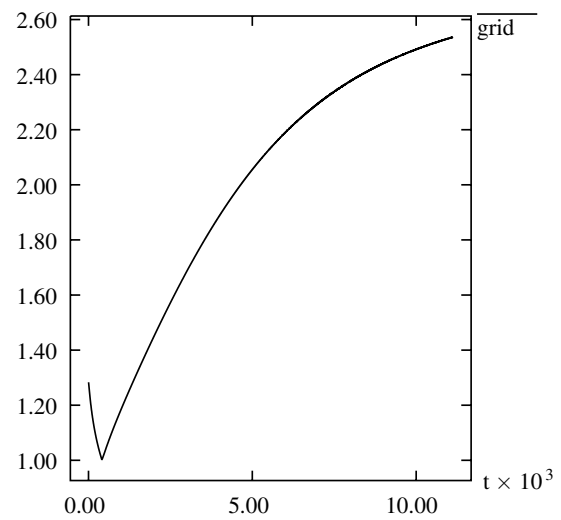

sigma

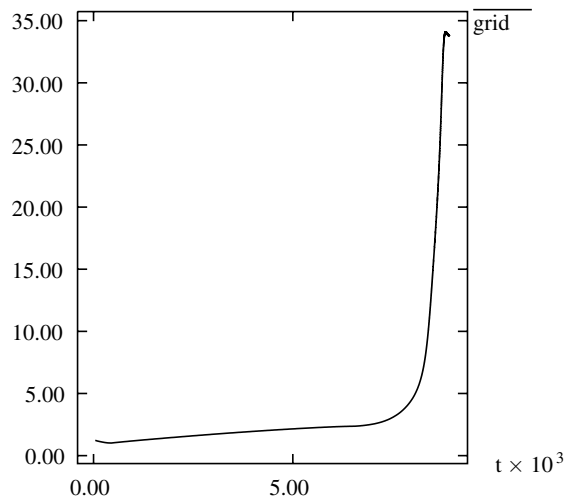

Figure 5. Value of the grid parameter $\sigma$ during the evolution for Example 4.3 for $\delta=0$ (left) and $\delta=0.1$ (right). 

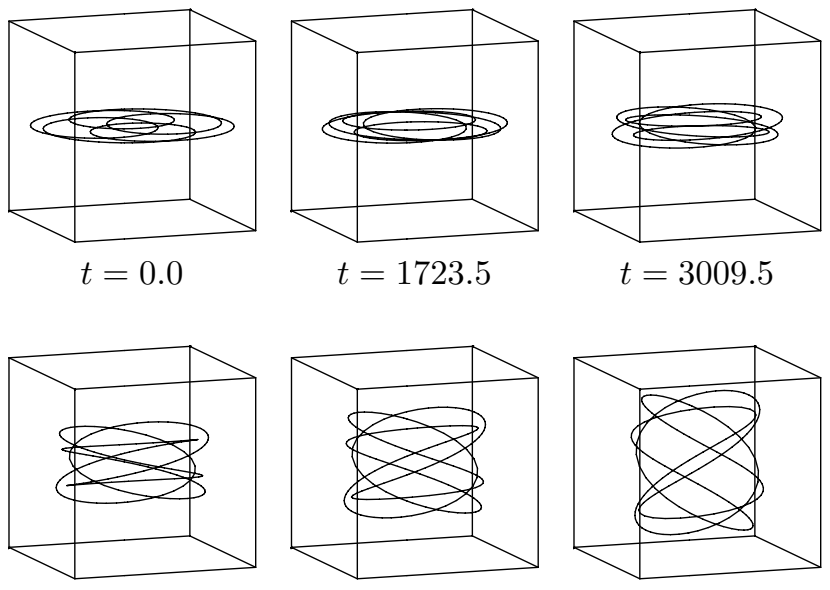

$t=4901.7$

$$
t=5664.6
$$

$t=.632 .5$
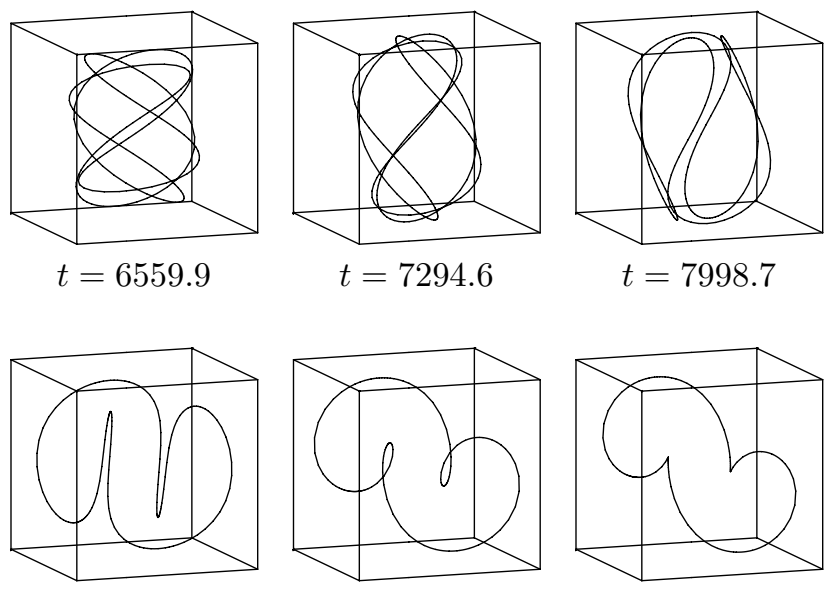

$t=8451.9$

$t=8666.3$

$t=8722.0$
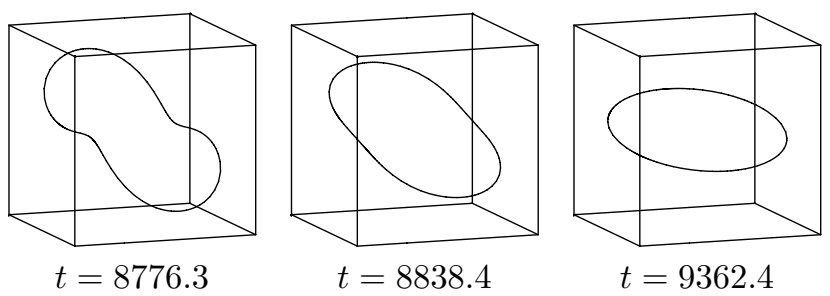

Figure 6. Example 4.3 for $\delta=0.1$ : evolution of a vertically perturbed hypocycloid towards a circle. 


\section{REFERENCES}

1. J.W. Barrett, H. Garcke, R. Nürnberg, A parametric finite element method for fourth order geometric evolution equations, J. Comput. Phys. 222(1) (2007), 441-467. MR 2298053 (2008a:65176)

2. K. Deckelnick, G. Dziuk, Error analysis of a finite element method for the Willmore flow of graphs, Interfaces Free Bound. 8 (2006), 21-46. MR2231251 (2007d:65083)

3. K. Deckelnick, G. Dziuk, C.M. Elliott, Computation of geometric partial differential equations and mean curvature flow, Acta Numer. 14 (2005), 139-232. MR2168343 (2006h:65159)

4. G. Dziuk, Computational parametric Willmore flow, Preprint Fakultät für Mathematik und Physik 07-13 (2007).

5. G. Dziuk, E. Kuwert, R. Schätzle, Evolution of elastic curves in $\mathbb{R}^{n}$ : Existence and computation, SIAM J. Math. Anal. 33 (2002), 1228-1245. MR.1897710 (2003f:53117)

6. A. Polden, Curves and surfaces of least total curvature and fourth-order flows, Ph.D. dissertation, Universität Tübingen, Tübingen, Germany, 1996.

Institut FÜr ANALYsis Und NUMERIK, OtTo-VON-Guericke-Universität Magdeburg, Universitätsplatz 2, 39106 Magdeburg, Germany

Abteilung für Angewandte Mathematik, Mathematisches Institut, Universität Freiburg, Hermann-Herder-Str. 10, 79104 Freiburg, Germany 